\title{
Bounds on treatment effects on transitions
}

\author{
Johan Vikström $^{\mathrm{a}, *}$, Geert Ridder ${ }^{\mathrm{b}}$, Martin Weidner ${ }^{\mathrm{c}, \mathrm{d}}$ \\ a IFAU-Uppsala and UCLS at Uppsala University, Box 513, 75120 Uppsala, Sweden \\ b Department of Economics, Kaprielian Hall, University of Southern California, Los Angeles, CA 90089, USA \\ ${ }^{\mathrm{c}}$ Department of Economics, University College London, Gower Street, London WC1E 6BT, UK \\ d CEMMAP, UK
}

\section{A R T I C L E I N F O}

\section{Article history:}

Received 12 January 2015

Received in revised form 27 September

2017

Accepted 22 November 2017

Available online 24 April 2018

\section{JEL classification:}

C14

C41

\section{Keywords:}

Partial identification

Duration model

Randomized experiment

Treatment effect

\begin{abstract}
A B S T R A C T
This paper considers the identification of treatment effects on conditional transition probabilities. We show that even under random assignment only the instantaneous average treatment effect is point identified. Since treated and control units drop out at different rates, randomization only ensures the comparability of treatment and controls at the time of randomization, so that long-run average treatment effects are not point identified. Instead, we derive bounds on these average effects. Our bounds do not impose (semi)parametric restrictions, for example, proportional hazards. We also explore assumptions such as monotone treatment response, common shocks and positively correlated outcomes that tighten the bounds.
\end{abstract}

(C) 2018 The Author(s). Published by Elsevier B.V. This is an open access article under the CC BY license (http://creativecommons.org/licenses/by/4.0/).

\section{Introduction}

We consider the effect of an intervention if the outcome is a transition from an initial to a destination state. The population of interest is a cohort of units that are in the initial state at the time zero. Treatment is assigned to a subset of the population either at the time zero or at some later time. Initially we assume that the treatment assignment is random. One main point made in this paper is that even if the treatment assignment is random, only certain average effects of the treatment are point identified. This is because the random assignment of treatment only ensures comparability of the treatment and control groups at the time of randomization. At later points in time treated units with characteristics that interact with the treatment to increase/decrease the transition probability relative to similar control units leave the initial state sooner/later than comparable control units, so that these characteristics are under/over represented among the remaining treated relative to the remaining controls and this confounds the effect of the treatment.

The confounding of the treatment effect through selective dropout is usually referred to as dynamic selection. Existing strategies that deal with dynamic selection rely heavily on parametric or semi-parametric model restrictions. An example is the approach of Abbring and Van den Berg (2003) who use the Mixed Proportional Hazard (MPH) model. In this model, the instantaneous transition or hazard rate is written as the product of a time effect, the effect of the intervention and an unobservable individual effect. As shown by Elbers and Ridder (1982), the MPH model is nonparametrically identified, so that if the multiplicative structure is maintained, identification does not rely on arbitrary functional form or distributional

\footnotetext{
* Corresponding author.

E-mail addresses: johan.vikstrom@ifau.uu.se (J. Vikström), ridder@usc.edu (G. Ridder), m.weidner@ucl.ac.uk (M. Weidner).
} 
assumptions beyond the assumed multiplicative specification. A second example is the approach of Heckman and Navarro (2007) who start from a threshold crossing model for transition probabilities. Again they establish semi-parametric identification, although their model requires the presence of additional covariates, besides the treatment indicator, that are independent of unobservable errors and have large support.

In this paper, we ask what can be identified if the identifying assumptions of the semi-parametric models do not hold. We show that, because of dynamic selection, we cannot point identify most average treatment effects of interest even under random assignment. However, we derive bounds on non-point-identified treatment effects, and show under what conditions they are informative. Our bounds are general, since beyond random assignment, we make no assumptions on functional form and additional covariates, and we allow for arbitrary heterogeneous treatment effects as well as arbitrary unobserved heterogeneity. The bounds can also be applied if the treatment assignment is unconfounded by creating bounds conditional on the covariates (or the propensity score) that are averaged over the distribution of these covariates (or the propensity score).

Besides these general bounds, we derive bounds under additional (weak) assumptions like monotone treatment response and positively correlated outcomes. We relate these assumptions to the assumptions made in the MPH model and to assumptions often made in discrete duration models and structural models. The additional assumptions often tighten the bounds considerably. We also discuss how to apply our various identification results to construct asymptotically valid confidence intervals for the respective treatment effects.

There are many applications in which we are interested in the effect of an intervention on transition probabilities/rates. The Cox (1972) partial likelihood estimator is routinely used to estimate the effect of an intervention on the survival rate of subjects. Transition models are used in several fields. Van den Berg (2001) surveys the models used and their applications. These models have also been used to study the effect of interventions on transitions. Examples are Ridder (1986), Card and Sullivan (1988), Bonnal et al. (1997), Gritz (1993), Ham and LaLonde (1996), Abbring and Van den Berg (2003), and Heckman and Navarro (2007). A survey of models for dynamic treatment effects can be found in Abbring and Heckman (2007).

An alternative to the effect of a treatment on the transition rate is its effect on the cdf of the time to transition or its inverse, the quantile function. This avoids the problem of dynamic selection. From the effect on the cdf we can recover the effect on the average duration, but we cannot obtain the effect on the conditional transition probabilities, so that the effect on the cdf is not informative on the evolution of the treatment effect over time. This is a limitation since there are good reasons as to why we should be interested in the effect of an intervention on the conditional transition probability or the transition/hazard rate. One important reason is the close link between the hazard rate and economic theory (Van den Berg (2001)). Economic theory often predicts how the hazard rate changes over time. For example, in the application to a job-bonus experiment considered in this paper, labor supply and search models predict that being eligible for a bonus if a job is found, increases the hazard rate from unemployment to employment. According to these models there is a positive effect only during the eligibility period, and the effect increases shortly before the end of the eligibility period. The timing of this increase depends on the arrival rate of job offers and is an indication of the control that the unemployed has over his/her re-employment time. Any such control has important policy implications. This can only be analyzed by considering how the effect on the hazard rate changes over time.

The evolution of the treatment effect over time is of key interest in different fields. For instance, consider two medical treatments that have the same effect on the average survival time. However, for one treatment the effect does not change over time while for the other the survival rate is initially low, e.g., due to side effects of the treatment, while after that initial period the survival rate is much higher. As another example, research on the effects of active labor market policies often documents a large negative lock-in effect and a later positive effect once the program has been completed, see e.g. the survey by Kluve et al. (2007).

We apply our bounds and confidence intervals to data from a job-bonus experiment previously analyzed by Meyer (1996) among others. As discussed above economic theory has specific predictions for the dynamic effect of a re-employment bonus with a finite eligibility period. Meyer (1996) estimates these dynamic effects using an MPH model. We study what can be identified if we rely solely on random assignment and some additional (weak) assumptions.

In Section 2 we define the treatment effects that are relevant if the outcome is a transition. Section 3 discusses their point or set identification in the case that the treatment is randomly assigned. This requires us to be precise on what we mean by random assignment in this setting. In Section 4 we explore additional assumptions that tighten the bounds. In Section 5 we derive the confidence intervals. Section 6 illustrates the bounds for the job-bonus experiment. Section 7 concludes.

\section{Setup}

\subsection{Motivating example}

In this paper we consider identification of the effect of a treatment on the conditional transition probability, usually referred to as the transition rate or the hazard rate. Effects on transition rates are important in many applications. The Illinois job-bonus experiment that we re-consider in the application in this paper is one example. The experiment that was conducted between mid-1984 and mid-1985 paid re-employment bonuses to unemployed individuals in the randomized treatment group who found employment within the first 11 weeks of unemployment. The fact that the bonus is only paid during the first 11 weeks has several interesting implications. Standard labor supply and search models predict that being 
eligible for the bonus should increase the transition rate from unemployment to employment during the 11 week eligibility period, but should have no effect after the end of the eligibility period. Another prediction is that the transition rate should increase shortly before the end of the eligibility period, as the unemployed run out of time to collect the bonus. These theoretical predictions can only be studied by examining how the effect of the job-bonus varies with time in unemployment, that is by studying the effect on the transition rate during the eligibility period, shortly before the end of the eligibility period and after the end of the eligibility period. Effects on the transition rate are also relevant in many other applications, including evaluations of medical treatments and active labor market policies.

The job-bonus experiment includes random treatment assignment, which ensures comparability of the treatment group and the control group at the time of randomization. At later time points some unemployed individuals have found a job, and this creates dynamic selection, that even under the initial random assignment might confound the comparability of the treatment and control groups. This is most easily seen if the fraction that has found a job differs between the two groups, and if those who have found a job have more favorable characteristics than those who remain unemployed. Under these conditions the remaining individuals in the treatment group will be negatively (positively) selected if the fraction remaining in unemployment is lower (higher) in the treatment group than in the control group. Moreover, even if the fraction still unemployed is the same in the treatment group and the control group we might still face a selection problem. In the jobbonus experiment, it could, for instance, be the case that individuals that respond to the bonus come from different parts of the ability distribution compared to those who find a job without the bonus. The implication of this is that the ability distribution differs between the treatment and the control groups, even if the fraction that has found a job is the same in the two groups. All this constitutes the dynamic selection problem that is addressed in this paper.

Previous studies that deal with the dynamic selection problem have mostly used parametric and semi-parametric models. For instance, Meyer (1996) uses a proportional hazard (PH) model to study how the effect of the job-bonus experiment considered in this paper varies before and after the 11 week eligibility period. A more general alternative to the PH model is to use a Mixed Proportional Hazard (MPH) model. In this model the instantaneous transition or hazard rate is written as the product of a time effect, the effect of the intervention and an unobservable individual effect. This model, however, imposes a multiplicative structure, a homogeneous treatment effect as well as other restrictions. In this paper we instead consider what can be identified if we rely solely on random assignment and do not impose the parametric restrictions that are implicit in the MPH model and other parametric and semi-parametric models.

\subsection{Average treatment effect on transitions}

We discuss the definition and identification of treatment effects on transition rates in discrete time with transitions occurring at times $t=1,2, \ldots{ }^{1}$ We assume that treatment is assigned at the beginning of the first period and that each unit is either always treated or always non-treated. In Section 3.1 we generalize these results by allowing the treatment to start in any time period. Let the potential outcome $Y_{t}^{1}$ be the indicator of a transition in period $t$ if treated and similarly $Y_{t}^{0}$ be the potential outcome if non-treated.

In any definition of the causal effect of a treatment on the transition rate we must account for the dynamic selection that was discussed in the previous subsection. If we do not specify a model for the transition rate we need to find another way to maintain the comparability of the treatment and control groups over time. The approach that we take in this paper is to consider average transition rates where the average is taken over the same population for both treated and controls (or in general for different treatment arms). We initially propose to average over the subpopulation of individuals who would have survived until time $t$ if treated. This is the analogue of the average effect on the treated considered in the static treatment effect literature. This leads to the following definition.

Definition 1. The causal effect on the transition probability of the treated survivors in $t$ is the Average Treatment Effect on Treated Survivors (ATETS) defined by

$$
\text { ATETS }_{t}=\mathbb{E}\left(Y_{t}^{1} \mid Y_{t-1}^{1}=0, \ldots, Y_{1}^{1}=0\right)-\mathbb{E}\left(Y_{t}^{0} \mid Y_{t-1}^{1}=0, \ldots, Y_{1}^{1}=0\right) .
$$

The differential selection only starts after the first period and the ATETS $S_{t}$ controls for that by comparing the transition rates for individuals with a common survival experience. ${ }^{2}$

Note that we are only concerned with the comparability of the treatment and control groups over the spell, i.e. with the different levels of dynamic selection in the two groups. If we keep the treatment and control groups comparable over time, there is still the question of how to interpret the time path of the average treatment effect over the spell. In this paper we do not try to decompose this path into the average treatment effect for a population of unchanging composition and a selection effect relative to this population. We do not define the treatment effect for this population of unchanging composition, but rather for a population with a composition that changes over time due to dynamic selection. The dynamic selection is made equal in the treatment and control groups, so that the treatment effect is not confounded by dynamic selection. Again this is analogous to the difference between the Average Treatment Effect and the Average Treatment Effect on the Treated in the case of a static treatment effect where the latter is defined for the population selected for treatment and the treatment effect is for this selective population.

\footnotetext{
1 The definition of causal effects in continuous time adds technical problems (see e.g. Gill and Robins (2001)) that would distract from the conceptual issues.

2 In Appendix C we also consider the average effect for the subpopulation of individuals who would have survived until $t$ under both treatment and no treatment.
} 
Table 1

For the case $T=2$ there are sixteen possible realizations for the potential outcomes $Y_{1}^{1}, Y_{2}^{1}, Y_{1}^{0}, Y_{2}^{0}$, and the corresponding probabilities are given in the table. The table also shows the row- and column-sums that are point identified from the data ("observable"). The four underlined probabilities are those that enter into the numerator of $\mathbb{E}\left(Y_{2}^{0} \mid Y_{1}^{1}=0\right)$, see Eq. (4) in the main text.

\begin{tabular}{|c|c|c|c|c|c|}
\hline & $Y^{0}=(0,0)$ & $Y^{0}=(0,1)$ & $Y^{0}=(1,0)$ & $Y^{0}=(1,1)$ & Observable (row sum) \\
\hline$Y^{1}=(0,0)$ & $p_{00,00}$ & $p_{00,01}$ & $p_{00,10}$ & $p_{00,11}$ & $\operatorname{Pr}(Y=(0,0) \mid D=1)$ \\
\hline$Y^{1}=(0,1)$ & $p_{01,00}$ & $\overline{p_{01,01}}$ & $p_{01,10}$ & $\overline{p_{01,11}}$ & \} $\operatorname{Pr}(Y=(0,1) \mid D=1)$ \\
\hline$Y^{1}=(1,0)$ & $p_{10,00}$ & $p_{10,01}$ & $p_{10,10}$ & $p_{10,11}$ & \multirow{2}{*}{$\operatorname{Pr}\left(Y_{1}=1 \mid D=1\right)$} \\
\hline$Y^{1}=(1,1)$ & $p_{11,00}$ & $p_{11,01}$ & $p_{11,10}$ & $p_{11,11}$ & \\
\hline $\begin{array}{l}\text { Observable } \\
\text { (col. sum) }\end{array}$ & $\operatorname{Pr}(Y=(0,0) \mid D=0)$ & $\operatorname{Pr}(Y=(0,1) \mid D=0)$ & \multicolumn{2}{|c|}{$\operatorname{Pr}\left(Y_{1}=1 \mid D=0\right)$} & \\
\hline
\end{tabular}

\section{Bounds on average treatment effects on transitions}

We now consider identification of the ATETS $t$ under random treatment assignment. Let $D$ be the indicator of treatment status, and $Y_{t}$ be the observed indicator of a transition in period $t$. The observed outcomes are related to the potential outcomes by the observation rule ${ }^{3}$

$$
Y_{t}=D Y_{t}^{1}+(1-D) Y_{t}^{0}
$$

We make the following random assignment assumption.

Assumption 1 (Random Assignment of Treatment).

$$
D \perp\left\{Y_{t}^{1}, Y_{t}^{0}: t=1,2, \ldots\right\} .
$$

In the first period $t=1$ no dynamic selection has taken place, yet, so subjects are fully randomized. Under Assumption 1 we therefore have the usual result, for $d \in\{0,1\}$,

$$
\mathbb{E}\left(Y_{1}^{d}\right)=\mathbb{E}\left(Y_{1} \mid D=d\right),
$$

implying that we can point identify the instantaneous treatment effect:

$$
\text { ATETS }_{1}=\mathbb{E}\left(Y_{1}^{1}\right)-\mathbb{E}\left(Y_{1}^{0}\right)=\mathbb{E}\left(Y_{1} \mid D=1\right)-\mathbb{E}\left(Y_{1} \mid D=0\right) .
$$

Next, we consider the identification of ATETS $t$ for $t=2$. We discuss this two period case in detail, because the main results of this paper can be understood in this two period setting, where the transition occurs in period 1, period 2 or after period 2. The two period dynamic treatment effect is defined by

$$
\operatorname{ATETS}_{2}=\mathbb{E}\left(Y_{2}^{1} \mid Y_{1}^{1}=0\right)-\mathbb{E}\left(Y_{2}^{0} \mid Y_{1}^{1}=0\right) .
$$

Under Assumption 1 we again have, for $d \in\{0,1\}$,

$$
\mathbb{E}\left(Y_{2}^{d} \mid Y_{1}^{d}=0\right)=\mathbb{E}\left(Y_{2} \mid Y_{1}=0, D=d\right) .
$$

Thus, the first term in ATETS ${ }_{2}$ is point identified from the data, and we can also point identify $\mathbb{E}\left(Y_{2}^{0} \mid Y_{1}^{0}=0\right)$. However, in this last expression the conditioning is on the survivors under non-treatment instead of under treatment, so this is not the second term in $\mathrm{ATETS}_{2}$. It turns out that $\mathbb{E}\left(Y_{2}^{0} \mid Y_{1}^{1}=0\right)$ is only partially identified from the data, and the goal in the following is therefore to derive bounds on this conditional expectation.

For every member of the population we have a vector of four binary potential outcomes $Y_{1}^{1}, Y_{2}^{1}, Y_{1}^{0}, Y_{2}^{0}$, for which there are $2^{4}=16$ possible realizations. We denote the probability of $\left(Y_{1}^{1}, Y_{2}^{1}, Y_{1}^{0}, Y_{2}^{0}\right)=\left(d_{1}, d_{2}, d_{3}, d_{4}\right)$ by $p_{d_{1} d_{2}, d_{3} d_{4}}$. Table 1 shows those sixteen population probabilities, using the two-vector notation $Y=\left(Y_{1}, Y_{2}\right)$ and $Y^{d}=\left(Y_{1}^{d}, Y_{2}^{d}\right)$. From the data we can identify the transition probabilities $\operatorname{Pr}\left(Y_{1}=1 \mid D=d\right)$ (transition in $t=1$ under treatment $d$ ), and $\operatorname{Pr}(Y=(0,1) \mid D=d)$ (transition in $t=2$ under treatment $d$ ), and $\operatorname{Pr}(Y=(0,0) \mid D=d)$ (transition after $t=2$ under treatment $d$ ). Those "observable" transition probabilities are obtained in Table 1 as row- and column-sums, for example we have $\operatorname{Pr}(Y=(0,0) \mid D=1)=p_{00,00}+p_{00,01}+p_{00,10}+p_{00,11}$.

Notice that $Y^{d}=(1,1)$ is included as a potential outcome here, that is, we allow for multiple transitions. Multiple transitions cannot occur if the destination state is absorbing, as in the job-bonus experiment. In that case we know that the probabilities in the last row and column of Table 1 are zero, that is,

$$
p_{11,00}=p_{11,01}=p_{11,10}=p_{11,10}=p_{11,11}=p_{00,11}=p_{01,11}=p_{10,11}=0 .
$$

\footnotetext{
3 In applications with at most a single transition per individual (where the destination state is absorbing), as in the job-bonus experiment, we have $\sum_{t} Y_{t} \leq 1$, but we still consider future $Y_{t}$ to be observed, even after the transition occurred.
} 
This information could sharpen the lower bound on the treatment effect, but we will not derive separate bounds for the case of an absorbing destination state. The bounds for the non-absorbing destination state are conservative if the destination is indeed absorbing.

With those definitions we obtain ${ }^{4}$

$$
\mathbb{E}\left(Y_{2}^{0} \mid Y_{1}^{1}=0\right)=\operatorname{Pr}\left(Y_{2}^{0}=1 \mid Y_{1}^{1}=0\right)=\frac{p_{00,01}+p_{00,11}+p_{01,01}+p_{01,11}}{\operatorname{Pr}\left(Y_{1}=0 \mid D=1\right)} .
$$

The denominator of the last expression is identified from the data. What is left to do is to provide bounds on the numerator in terms of the six observable transition probabilities $\operatorname{Pr}\left(Y_{1}=1 \mid D=d\right), \operatorname{Pr}(Y=(0,1) \mid D=d)$, and $\operatorname{Pr}(Y=(0,0) \mid D=d)$, $d \in\{0,1\}$. The four probabilities that enter into this numerator are underlined in Table 1 .

Thus, the question is what values for $p_{00,01}+p_{00,11}+p_{01,01}+p_{01,11}$ are feasible, subject to the positivity condition $p_{d_{1} d_{2}, d_{3} d_{4}} \geq 0$ for all $\left(d_{1}, d_{2}, d_{3}, d_{3}\right) \in\{0,1\}^{4}$, and subject to the constraint that the row- and column sums in Table 1 equal to the six observable transition probabilities. Two upper bounds are given by

$$
\begin{aligned}
& p_{00,01}+p_{00,11}+p_{01,01}+p_{01,11} \leq 1-\operatorname{Pr}\left(Y_{1}=1 \mid D=1\right), \\
& p_{00,01}+p_{00,11}+p_{01,01}+p_{01,11} \leq 1-\operatorname{Pr}(Y=(0,0) \mid D=0) .
\end{aligned}
$$

Here, the first upper bound follows from the row-sum conditions in Table 1, which require that $p_{00,01}+p_{00,11}+p_{01,01}+p_{01,11}$ is smaller than $\operatorname{Pr}(Y=(0,0) \mid D=1)+\operatorname{Pr}(Y=(0,1) \mid D=1)$, which equals $1-\operatorname{Pr}\left(Y_{1}=1 \mid D=1\right)$. Analogously, the second upper bound follows from the column-sum conditions in Table 1 , which require that $p_{00,01}+p_{00,11}+p_{01,01}+p_{01,11}$ is smaller than $\operatorname{Pr}(Y=(0,1) \mid D=0)+\operatorname{Pr}\left(Y_{1}=1 \mid D=0\right)$, which equals $1-\operatorname{Pr}(Y=(0,0) \mid D=0)$. Note that if the destination state is absorbing, the second upper bound in $(5)$ is $\operatorname{Pr}(Y=(0,1) \mid D=0)$ which is smaller than for the non-absorbing case.

Regarding the lower bound, notice that $p_{00,01}+p_{01,01}$ cannot be arbitrarily small, because when shifting probability mass within the column of Table 1 that corresponds to $\operatorname{Pr}(Y=(0,1) \mid D=0)$, we cannot increase the other elements in this column (i.e. $\left.p_{10,01}+p_{11,01}\right)$ to more than $\operatorname{Pr}\left(Y_{1}=1 \mid D=1\right)$, since we would otherwise violate the corresponding row-constraint. By that argument we find the bound $p_{00,01}+p_{01,01} \geq \operatorname{Pr}(Y=(0,1) \mid D=0)-\operatorname{Pr}\left(Y_{1}=1 \mid D=1\right)$. Together with the positivity condition on all probabilities we thus obtain

$$
p_{00,01}+p_{00,11}+p_{01,01}+p_{01,11} \geq \max \left\{0, \operatorname{Pr}(Y=(0,1) \mid D=0)-\operatorname{Pr}\left(Y_{1}=1 \mid D=1\right)\right\} .
$$

The lower bound is the same if the destination state is absorbing. Combining (3)-(6), gives the bounds on ATETS 2 summarized in the following theorem. ${ }^{5}$ We find it convenient to present the theorem for the case of ATETS $t_{t}$ for arbitrary $t$. For this we introduce the notation $\bar{Y}_{t-1}=\left(Y_{1}, \ldots, Y_{t-1}\right)$, and we write 0 for the vector of zeros.

Theorem 1 (Bounds on ATETS). Suppose that Assumption 1 holds. Let $t \in\{2,3,4, \ldots\}$. If $\operatorname{Pr}\left(\bar{Y}_{t-1}=0 \mid D=1\right)=0$, then ATETS $_{t}$ is not defined. If $\operatorname{Pr}\left(\bar{Y}_{t-1}=0 \mid D=1\right)>0$, and also $\operatorname{Pr}(D=1)>0$ and $\operatorname{Pr}(D=0)>0$, then we have the bounds

$$
\mathrm{LB}_{t} \leq \mathrm{ATETS}_{t} \leq \mathrm{UB}_{t},
$$

where

$$
\begin{aligned}
\mathrm{LB}_{t} \equiv & \operatorname{Pr}\left(Y_{t}=1 \mid \bar{Y}_{t-1}=0, D=1\right) \\
& -\min \left\{1, \frac{1-\left[1-\operatorname{Pr}\left(Y_{t}=1 \mid \bar{Y}_{t-1}=0, D=0\right)\right] \operatorname{Pr}\left(\bar{Y}_{t-1}=0 \mid D=0\right)}{\operatorname{Pr}\left(\bar{Y}_{t-1}=0 \mid D=1\right)}\right\}, \\
\mathrm{UB}_{t} \equiv \operatorname{Pr}\left(Y_{t}=1 \mid \bar{Y}_{t-1}=0, D=1\right) & \\
& -\max \left\{0, \frac{\operatorname{Pr}\left(Y_{t}=1 \mid \bar{Y}_{t-1}=0, D=0\right) \operatorname{Pr}\left(\bar{Y}_{t-1}=0 \mid D=0\right)-1}{\operatorname{Pr}\left(\bar{Y}_{t-1}=0 \mid D=1\right)}+1\right\} .
\end{aligned}
$$

Proof. See Appendix A.

$$
\begin{aligned}
& { }^{4} \text { We have } \\
& \qquad \operatorname{Pr}\left(Y_{2}^{0}=1 \mid Y_{1}^{1}=0\right)=\frac{\operatorname{Pr}\left(Y_{2}^{0}=1 \& Y_{1}^{1}=0\right)}{\operatorname{Pr}\left(Y_{1}^{1}=0\right)}=\frac{\operatorname{Pr}\left\{\left[Y^{0}=(0,1) \text { or }(1,1)\right] \&\left[Y^{1}=(0,0) \text { or }(0,1)\right]\right\}}{\operatorname{Pr}\left(Y_{1}=0 \mid D=1\right)},
\end{aligned}
$$

where we also used the random assignment assumption.

5 Combining (4)-(6) and $\operatorname{Pr}\left(Y_{1}=1 \mid D=1\right)=1-\operatorname{Pr}\left(Y_{1}=0 \mid D=1\right)$ we obtain

$$
\max \left\{0, \frac{\operatorname{Pr}(Y=(0,1) \mid D=0)-1}{\operatorname{Pr}\left(Y_{1}=0 \mid D=1\right)}+1\right\} \leq \mathbb{E}\left(Y_{2}^{0} \mid Y_{1}^{1}=0\right) \leq \min \left\{1, \frac{1-\operatorname{Pr}(Y=(0,0) \mid D=0)}{\operatorname{Pr}\left(Y_{1}=0 \mid D=1\right)}\right\} .
$$

Also using (3) and $\mathbb{E}\left(Y_{2}^{1} \mid Y_{1}^{1}=0\right)=\operatorname{Pr}\left(Y_{2}=1 \mid Y_{1}=0, D=1\right)$, and rewriting $\operatorname{Pr}(Y=(0,1) \mid D=0)$ and $\operatorname{Pr}(Y=(0,0) \mid D=0)$ as products of one-step ahead conditional transition probabilities gives the bounds in Theorem 1 for the case $t=2$. 
Notice that the bounds in Theorem 1 require no assumptions beyond random assignment. They allow, for instance, for arbitrary heterogeneity in treatment response. The bounds exist as long as $\operatorname{Pr}\left(\bar{Y}_{t-1}=0 \mid D=1\right)>0$, because if this probability is zero, then the subpopulation for which ATETS $_{t}$ is defined has no members. ${ }^{6}$ The conditions $\operatorname{Pr}(D=1)>0$ and $\operatorname{Pr}(D=0)>0$ guarantee that both treated and untreated individuals are observed, which is an obvious condition for any treatment effect estimation.

Next, consider the intuition behind these bounds using the job-bonus experiment as an illustration. Both the upper and the lower bound are increasing in the observed transition probability from unemployment to employment in the treatment group in period $t, \operatorname{Pr}\left(Y_{t}=1 \mid \bar{Y}_{t-1}=0, D=1\right)$. This follows directly from the fact that we consider the average effect for treated individuals that remain in unemployment until time $t$. The bounds also depend on the observed transition probability in the control group, $\operatorname{Pr}\left(Y_{t}=1 \mid \bar{Y}_{t-1}=0, D=0\right)$, but this relationship is more complicated than the relationship between the bounds and $\operatorname{Pr}\left(Y_{t}=1 \mid \bar{Y}_{t-1}=0, D=1\right)$. In general we have that both the upper and the lower bound are decreasing in $\operatorname{Pr}\left(Y_{t}=1 \mid \bar{Y}_{t-1}=0, D=0\right)$. The reason for this is that a high transition rate among the unemployed individuals in the control group allows for a larger counterfactual outcome under no treatment. Another important determinant of the bounds is the fraction in the treatment group that remains in unemployment until time $t, \operatorname{Pr}\left(\bar{Y}_{t-1}=0 \mid D=1\right)$. If this survival probability is small, there is more selection in the group of treated that remains in unemployment, i.e. more pronounced dynamic selection, leading to a larger difference between the upper and the lower bound.

From Theorem 1 we also have several other implications. Corollary 1 shows that if the survival rates under treatment and control both equal one, i.e., if $\operatorname{Pr}\left(\bar{Y}_{t-1}=0 \mid D=0\right)=1$ and $\operatorname{Pr}\left(\bar{Y}_{t-1}=0 \mid D=1\right)=1$, then the dynamic treatment effect ATETS $_{t}$ is point identified. If everyone survives the first $t-1$ periods we have under random treatment assignment in period 1 two groups of equal composition even in period $t$.

Corollary 1 (Point Identification). ATETS ${ }_{t}$ is point identified if both $\operatorname{Pr}\left(\bar{Y}_{t-1}=0 \mid D=0\right)=1$ and $\operatorname{Pr}\left(\bar{Y}_{t-1}=0 \mid D=1\right)=1$.

The information in the bounds depends on the width of the implied interval. The best case is that the restrictions imposed by the max and $\min$ in $\mathrm{LB}_{t}$ and $\mathrm{UB}_{t}$ above are non-binding, and the width of the bounds then becomes

$$
\mathrm{UB}_{t}-\mathrm{LB}_{t}=\frac{2-\operatorname{Pr}\left(\bar{Y}_{t-1}=0 \mid D=1\right)-\operatorname{Pr}\left(\bar{Y}_{t-1}=0 \mid D=0\right)}{\operatorname{Pr}\left(\bar{Y}_{t-1}=0 \mid D=1\right)} .
$$

This expression shows that the width of the bound is decreasing in $\operatorname{Pr}\left(\bar{Y}_{t-1}=0 \mid D=1\right)$ and $\operatorname{Pr}\left(\bar{Y}_{t-1}=0 \mid D=0\right)$. In the job-bonus application this implies that the width of the bound is directly related to the probability that unemployed individuals in the treatment group and in the control group remain in unemployment until time $t$.

\subsection{Arbitrary time to treatment}

So far we have considered the case with treatment assignment at the beginning of the first period. We now consider a more general case in which the treatment can start in any time period. We assume that any treated unit remains treated in the subsequent periods, that is, we assume that treatment is an absorbing state. Let the potential outcome $Y_{t}^{k}$ be an indicator of a transition in period $t$ if the treatment started in period $k \leq t$, and since treatment is assumed to be an absorbing state this means that the unit is treated in all subsequent periods. The potential outcome if non-treated is denoted by $Y_{t}^{0} .{ }^{7}$

Let $D_{t}$ be the indicator of treatment in period $t$ so that a unit with $D_{t}=1$ could either be treated or non-treated before $t$. We use the notation $\bar{D}_{t-1}=\left(D_{1}, \ldots, D_{t-1}\right)$ and write 1 and 0 for the vector of ones and zeros. Note that because the treatment state is absorbing we have that $\bar{D}_{t-1}=1 \Leftrightarrow \bar{D}_{t}=1$.

With treatment assignments in all periods we need a different randomization assumption. The relevant random assignment assumption is

Assumption 2 (Sequential Randomization Among Survivors). For all $t$,

$$
D_{t} \perp\left\{Y_{s}^{k}: k, s=t, t+1, t+2, \ldots\right\} \mid \bar{D}_{t-1}=0, Y_{t-1}^{0}=\cdots=Y_{1}^{0}=0 .
$$

This assumption implies that treatment is assigned randomly among survivors that have not been treated before. ${ }^{8}$

\footnotetext{
6 The bounds in Theorem 1 also involve conditioning on the event $\bar{Y}_{t-1}=0$ and $D=0$, but we do not need to impose $\operatorname{Pr}\left(\bar{Y}_{t-1}=0 \mid D=0\right)>0$, because all expressions involving that conditioning set can be rewritten, for example, we have $\left[1-\operatorname{Pr}\left(Y_{t}=1 \mid \bar{Y}_{t-1}=0, D=0\right)\right] \operatorname{Pr}\left(\bar{Y}_{t-1}=0 \mid D=0\right)=$ $\operatorname{Pr}\left(Y_{t}=0 \& \bar{Y}_{t-1}=0 \mid D=0\right)$.

7 Under the no-anticipation assumption in Abbring and Van den Berg (2003), $Y_{t}^{0}$ corresponds to the potential outcome if never-treated, since noanticipation assures that the non-treated potential outcome at $t$ equals the potential outcome at $t$ if never-treated. Without the no-anticipation assumption the potential outcome, $Y_{t}^{0}$, corresponds to the potential outcome if non-treated up until $t$, including any anticipatory responses to information about treatments after $t$.

8 Sequential randomization occurs in medical studies in the Sequential Multiple Assignment Randomized Trial (SMART) design, see Murphy and Bingham (2009) and Murphy (2005).
} 
The treatment effect at $t$ of a treatment started in $k \leq t$ is ${ }^{9}$

$$
\operatorname{ATETS}_{t}(k)=\mathbb{E}\left(Y_{t}^{k} \mid \bar{Y}_{t-1}^{k}=0, \bar{D}_{k-1}=0\right)-\mathbb{E}\left(Y_{t}^{0} \mid \bar{Y}_{t-1}^{k}=0, \bar{D}_{k-1}=0\right) .
$$

where we average over the subpopulation that started treatment in $k$ and was not treated before $k$. For the instantaneous treatment effect (if there is no anticipation effect, the outcome in $k-1$ and earlier is the non-treated outcome)

$$
\operatorname{ATETS}_{k}(k)=\mathbb{E}\left(Y_{k}^{k} \mid \bar{Y}_{k-1}^{0}=0, \bar{D}_{k-1}=0\right)-\mathbb{E}\left(Y_{k}^{0} \mid \bar{Y}_{k-1}^{0}=0, \bar{D}_{k-1}=0\right) \text {. }
$$

Under sequential randomization as in Assumption 2 we have

$$
\begin{aligned}
& \operatorname{ATETS}_{k}(k)=\mathbb{E}\left(Y_{k}^{k} \mid \bar{Y}_{k-1}^{0}=0, D_{k}=1, \bar{D}_{k-1}=0\right)-\mathbb{E}\left(Y_{k}^{0} \mid \bar{Y}_{k-1}^{0}=0, D_{k}=0, \bar{D}_{k-1}=0\right)= \\
& \mathbb{E}\left(Y_{k} \mid \bar{Y}_{k-1}=0, D_{k}=1, \bar{D}_{k-1}=0\right)-\mathbb{E}\left(Y_{k} \mid \bar{Y}_{k-1}=0, D_{k}=0, \bar{D}_{k-1}=0\right)
\end{aligned}
$$

so that the instantaneous effect of a treatment starting at $k$ is point identified.

For the $\operatorname{ATETS}_{t}(k)$ in $(7)$ we derive the bounds as in Theorem 1 with $k$ the first period and time of randomization, i.e. in the role of period 1, and at time $k$ we consider the observations with $\bar{Y}_{k-1}^{0}=0, \bar{D}_{k-1}=0$, i.e. the survivors if not treated that did not receive treatment before $k$ which is the same as $\bar{Y}_{k-1}=0, \bar{D}_{k-1}=0$. For this subpopulation the data that enter the bounds are the transition probabilities in $t$ given treatment starting in $k$ (and continuing until $t$ ) and given being assigned to the control group at $k$ (and remaining in the control group until $t$ ). The bounds of Theorem 1 apply directly with obvious changes in the conditioning sets of the probabilities (condition on $\bar{Y}_{k-1}=0, \bar{D}_{k-1}=0$ in addition to the conditioning variables in the bounds of Theorem 1).

\section{Bounds on treatment effects on transitions under additional assumptions}

The bounds in the previous section did not impose any assumptions beyond random assignment. In this section, we explore the identifying power of additional assumptions. For sake of presentation we will focus on identification of ATETS ${ }_{t}{ }^{10}$ The assumptions that we make are implicit in parametric models such as the MPH model, and also in the discrete duration models and structural models presented in this section.

As a background consider the following discrete duration model for the control and treated outcomes, for individual $i$ in period $t$,

$$
\begin{aligned}
& Y_{i t}^{0}=I\left(\alpha_{t}+V_{i}-\varepsilon_{i t}^{0} \geq 0\right), \\
& Y_{i t}^{1}=I\left(\alpha_{t}+\gamma_{i t}+V_{i}-\varepsilon_{i t}^{1} \geq 0\right) .
\end{aligned}
$$

This discrete duration model has a composite error that is the sum of unobserved heterogeneity $V_{i}$ and a random shock $\varepsilon_{i t}$. Here, $\alpha_{t}$ is a time specific effect, and $\gamma_{i t}$ drives the systematic differences between treated and non-treated outcomes. The model allows for different random shocks under control, $\varepsilon_{i t}^{0}$ and treatment, $\varepsilon_{i t}^{1}$. These random shocks are assumed to be independent, but even in this case the potential outcomes are positively correlated through their dependence on $V_{i}$. A more traditional model has the same random shock under control and treatment, $\varepsilon_{i t}$, but this is a more restrictive model. In the sequel we start from the more general model in (8) to illustrate the additional assumptions.

\subsection{Monotone treatment response}

The first assumption is Monotone Treatment Response (MTR). The assumption is that the effect is either positive or negative for all units in all periods. In terms of the discrete duration model example in (8), the assumption is that $\gamma_{i t} \leq 0$ for all $i, t$ or $\gamma_{i t} \geq 0$ for all $i, t$. That is, we do not assume a specific direction of the effect, merely that the effect goes in the same direction for all units. For the job-bonus experiment considered in this paper this assumption rules out that the bonus offer increases the transition rate for some unemployed individuals and decreases the transition rate for others. The assumption is similar to the MTR assumption introduced by Manski (1997) and Manski and Pepper (2000).

To formally define MTR in our framework we denote the event of survival under treatment and no-treatment by $S_{t}$, that is, $S_{t}$ is the event that $\bar{Y}_{t}^{1}=0$ and $\bar{Y}_{t}^{0}=0$. We have

Assumption 3 (Monotone Treatment Response (MTR)). Either

$$
\operatorname{Pr}\left(Y_{t}^{1}=1 \mid S_{t-1}, V\right) \geq \operatorname{Pr}\left(Y_{t}^{0}=1 \mid S_{t-1}, V\right)
$$

\footnotetext{
9 Note that $\operatorname{ATETS}_{t}(k)$ with $k=1$ is identical to ATETS ${ }_{t}$ considered above. Here, we use the more general notation, ATETS $_{t}(k)$, to define the average effects when treatment could start in any period.

10 Assumptions that tighten $\operatorname{ATETS}_{t}(k)$ with $k>1$ follow using similar reasoning.
} 
for all $t$, or

$$
\operatorname{Pr}\left(Y_{t}^{1}=1 \mid S_{t-1}, V\right) \leq \operatorname{Pr}\left(Y_{t}^{0}=1 \mid S_{t-1}, V\right)
$$

for all $t$. Here, $V$ can be any known or unknown vector of individual specific characteristics (both observed and unobserved) that are constant over time. ${ }^{11}$

This assumption can be relaxed at the expense of more complicated bounds. The assumption is that the effect goes in the same direction for all units. This is consistent with a discrete duration model that allows the random and independent $\operatorname{shocks} \varepsilon_{i t}^{1}$ and $\varepsilon_{i t}^{0}$ to differ, but restricts the sign of $\gamma_{i t}$.

\subsection{Common shocks}

The next assumption restricts the joint distribution of potential outcomes in the treatment arms. The assumption essentially imposes that the outcomes in both treatment arms involve the same random shock. In terms of the discrete duration model example in (8), the assumption is that $\varepsilon_{i t}^{1}=\varepsilon_{i t}^{0}=\varepsilon_{i t}$, so that the random shock $\varepsilon_{i t}$ is the same for both treatment states. Thus, if $\gamma_{i t} \leq 0$ then the treated have a larger survival probability in $t$. Therefore the event that $i$ survives in $t$ if not treated, i.e. $Y_{i t}^{0}=0$, is equivalent to $\varepsilon_{i t} \geq \alpha_{t}+V_{i}$, so that this event implies that $\varepsilon_{i t} \geq \alpha_{t}+\gamma_{i t}+V_{i} \geq 0$, i.e. $Y_{i t}^{1}=0$. In a structural model the random shocks often satisfy this restrictions, as is illustrated in a simple job search model below.

The formal statement of the assumption is as follows.

\section{Assumption 4 (Common Shocks (CS)). For all $t$}

$$
\begin{aligned}
& \operatorname{Pr}\left(Y_{t}^{1}=0 \mid S_{t-1}, V\right) \geq \operatorname{Pr}\left(Y_{t}^{0}=0 \mid S_{t-1}, V\right) \quad \Rightarrow \quad \operatorname{Pr}\left(Y_{t}^{1}=0 \mid S_{t-1}, Y_{t}^{0}=0, V\right)=1, \\
& \operatorname{Pr}\left(Y_{t}^{1}=0 \mid S_{t-1}, V\right) \leq \operatorname{Pr}\left(Y_{t}^{0}=0 \mid S_{t-1}, V\right) \quad \Rightarrow \quad \operatorname{Pr}\left(Y_{t}^{0}=0 \mid S_{t-1}, Y_{t}^{1}=0, V\right)=1 .
\end{aligned}
$$

Here, again, $V$ can be any known or unknown vector of individual specific characteristics (both observed and unobserved) that are constant over time. ${ }^{12}$

In the job-bonus application the intuition behind this assumption is that CS implies that all random events leading to a job offer and employment are the same irrespective if a specific unemployed individual is randomized to the treatment group or to the control group.

Assumption 4 is satisfied in many standard structural models. Consider for instance a non-stationary job search model for an unemployed individual as in Van den Berg (1990) or Meyer (1996). The treatment is a re-employment bonus as discussed in Section 5 below. In each period a job offer is obtained with probability $p\left(t, V_{i}\right)$. Let $Y_{\text {of }, \text { it }}$ be the indicator of an offer in period $t$ and $Y_{o f, i t}=I\left(\varepsilon_{o f, i t} \in A\left(t, V_{i}\right)\right)$ with $A\left(t, V_{i}\right)$ a set. If the job offer is not under control of $i$, the arrival process is the same under treatment and control. The reservation wage is denoted by $\xi_{i t}^{1}$ for the treated and $\xi_{i t}^{0}$ for the controls. In general (see Meyer (1996) ) $\xi^{1}\left(t, V_{i}\right) \leq \xi^{0}\left(t, V_{i}\right)$, so that if $H$ is the wage offer c.d.f. we have the acceptance probabilities $1-H\left(\xi^{1}\left(t, V_{i}\right)\right) \geq 1-H\left(\xi^{0}\left(t, V_{i}\right)\right)$. The acceptance indicators are $Y_{a c, i t}^{0}=I\left(\varepsilon_{w, i t} \geq \xi^{0}\left(t, V_{i}\right)\right)$ and $Y_{a c, i t}^{1}=I\left(\varepsilon_{w, i t} \geq \xi^{1}\left(t, V_{i}\right)\right)$ with $\varepsilon_{w, i t}$ the wage offer. Because $Y_{i t}^{0}=Y_{o f, i t} Y_{a c, i t}^{0}$ and $Y_{i t}^{1}=Y_{o f, i t} Y_{a c, i t}^{1}$, we have

$$
Y_{i t}^{1}=0 \Rightarrow Y_{i t}^{0}=0,
$$

so that Assumption 4 is satisfied.

\subsection{Positively correlated outcomes}

The third assumption concerns the relation between the counterfactual outcomes over time. Let us introduce the assumption for the two period case. If we compare the transition probability $\operatorname{Pr}\left(Y_{2}^{0}=1 \mid Y_{1}^{1}=0, Y_{1}^{0}=0\right)$ to $\operatorname{Pr}\left(Y_{2}^{0}=1 \mid Y_{1}^{1}=\right.$ $1, Y_{1}^{0}=0$ ), i.e. the probability of a transition in period 2 if no treatment was received in periods 1 and 2 given survival with or without treatment in period 1 to the same probability given survival without but not with treatment in period 1 , then it is reasonable to assume that the former probability is not larger than the latter. Individuals with $Y_{1}^{1}=0, Y_{1}^{0}=0$ have characteristics that make them not leave the initial state as opposed to individuals with $Y_{1}^{1}=1, Y_{1}^{0}=0$ that have characteristics that make them leave the initial state if treated in period 1 . If the variables that affect the transition out of the initial state are positively correlated between periods, then

$$
\operatorname{Pr}\left(Y_{2}^{0}=1 \mid Y_{1}^{1}=0, Y_{1}^{0}=0\right) \leq \operatorname{Pr}\left(Y_{2}^{0}=1 \mid Y_{1}^{1}=1, Y_{1}^{0}=0\right)
$$

\footnotetext{
11 In particular, $V$ could identify the individual $i$ uniquely. In that case the assumption simply becomes that we have either $\operatorname{Pr}\left(Y_{i t}^{1}=1 \mid S_{i, t-1}\right) \geq$ $\operatorname{Pr}\left(Y_{i t}^{0}=1 \mid S_{i, t-1}\right)$, for all $i, t$, or $\operatorname{Pr}\left(Y_{i t}^{1}=1 \mid S_{i, t-1}\right) \leq \operatorname{Pr}\left(Y_{i t}^{0}=1 \mid S_{i, t-1}\right)$, for all $i$, t. This was the formulation of the assumption used in a previous version of this paper.

12 In particular, $V$ could identify the individual $i$ uniquely. In that case the assumption simply becomes that for $i$ we have $\operatorname{Pr}\left(Y_{i t}^{1}=0 \mid S_{i, t-1}\right) \geq \operatorname{Pr}\left(Y_{i t}^{0}=\right.$ $\left.0 \mid S_{i, t-1}\right) \quad \Rightarrow \quad \operatorname{Pr}\left(Y_{i t}^{1}=0 \mid S_{i, t-1}, Y_{i t}^{0}=0\right)=1$, and $\operatorname{Pr}\left(Y_{i t}^{1}=0 \mid S_{i, t-1}\right) \leq \operatorname{Pr}\left(Y_{i t}^{0}=0 \mid S_{i, t-1}\right) \quad \Rightarrow \quad \operatorname{Pr}\left(Y_{i t}^{0}=0 \mid S_{i, t-1}, Y_{i t}^{1}=0\right)=1$.
} 
As before we motivate the assumption using the discrete duration model in (8). Consider (9). By the discrete duration model the conditioning events are if no transition (i.e., if $Y_{1}^{1}=0, Y_{1}^{0}=0$ )

$$
V_{i}-\varepsilon_{i 1}^{0}<-\alpha_{1}, V_{i}-\varepsilon_{i 1}^{1}<-\alpha_{1}-\gamma_{i 1},
$$

and if a transition in 1 if treated (i.e., if $Y_{1}^{1}=1, Y_{1}^{0}=0$ )

$$
V_{i}-\varepsilon_{i 1}^{0}<-\alpha_{1}, V_{i}-\varepsilon_{i 1}^{1} \geq-\alpha_{1}-\gamma_{i 1} .
$$

Thus, if $V_{i}-\varepsilon_{i 1}^{0}$ is positively correlated with $V_{i}-\varepsilon_{i 2}^{0}$, then (9) holds, since then $\operatorname{Pr}\left(Y_{2}^{0}=1\right)$ is at least as large for the subpopulation with $Y_{1}^{1}=0, Y_{1}^{0}=0$ as for the subpopulation with $Y_{1}^{1}=1, Y_{1}^{0}=0$. We call this positively correlated outcomes. An analogous argument can be made for the relation between $\operatorname{Pr}\left(Y_{2}^{0}=1 \mid Y_{1}^{1}=0, Y_{1}^{0}=1\right)$ and $\operatorname{Pr}\left(Y_{2}^{0}=1 \mid Y_{1}^{1}=0, Y_{1}^{0}=0\right)$, as well as for $\operatorname{Pr}\left(Y_{2}^{1}=1\right)$ for different subpopulations.

Formally, for arbitrary $t$ we have

Assumption 5 (Positively Correlated Outcomes (PCO)). For all $m=1, \ldots, t-1$

$$
\begin{aligned}
& \operatorname{Pr}\left(Y_{t}^{0}=1 \mid Y_{m}^{1}=1, \bar{Y}_{m-1}^{1}=0, \bar{Y}_{t-1}^{0}=0\right) \geq \operatorname{Pr}\left(Y_{t}^{0}=1 \mid \bar{Y}_{t-1}^{1}=0, \bar{Y}_{t-1}^{0}=0\right), \\
& \operatorname{Pr}\left(Y_{t}^{1}=1 \mid Y_{m}^{1}=1, \bar{Y}_{m-1}^{1}=0, \bar{Y}_{t-1}^{0}=0\right) \geq \operatorname{Pr}\left(Y_{t}^{1}=1 \mid \bar{Y}_{t-1}^{1}=0, \bar{Y}_{t-1}^{0}=0\right), \\
& \operatorname{Pr}\left(Y_{t}^{0}=1 \mid \bar{Y}_{t-1}^{1}=0, Y_{m}^{0}=1, \bar{Y}_{m-1}^{0}=0\right) \geq \operatorname{Pr}\left(Y_{t}^{0}=1 \mid \bar{Y}_{t-1}^{1}=0, \bar{Y}_{t-1}^{0}=0\right), \\
& \operatorname{Pr}\left(Y_{t}^{1}=1 \mid \bar{Y}_{t-1}^{1}=0, Y_{m}^{0}=1, \bar{Y}_{m-1}^{0}=0\right) \geq \operatorname{Pr}\left(Y_{t}^{1}=1 \mid \bar{Y}_{t-1}^{1}=0, \bar{Y}_{t-1}^{0}=0\right) .
\end{aligned}
$$

For the job-bonus application PCO has several implications. As an illustration, consider two groups consisting of unemployed who find and unemployed who do not find employment in the first period if non-treated. In this case PCO implies that in the second period, the transition rate under treatment on average is weakly larger in the former group compared to the latter. This holds if the ranking of the unemployed individuals in terms of the characteristics that determine job offers, such as ability, experience and job search effort, remains the same during the entire unemployment spell.

Note that the motivating example above shows that PCO does not imply nor is implied by MTR or CS. The CS assumption is on the contemporaneous correlation of random shocks while PCO relates to a (positive) relation of the combined random error over time. Since the latter in general contains an important individual effect, positive correlation is not a strong assumption.

\subsection{Bounds under the additional assumptions}

We now obtain bounds on ATETS for arbitrary $t$ when we compare a treatment started in period 1 to no treatment in all periods. Bounds under MTR and CS are given in Theorems 2 and 3 provides bounds under PCO. Bounds under all three additional assumptions are in Theorem 4.

Theorem 2 (Bounds on ATETS Under MTR and CS for $t$ Periods). Let Assumptions 1,3 and 4 hold. Let $t \in\{2,3,4, \ldots\}$. If $\operatorname{Pr}\left(\bar{Y}_{t-1}=0 \mid D=1\right)=0$, then ATETS $_{t}$ is not defined.

If $\operatorname{Pr}\left(\bar{Y}_{t-1}=0 \mid D=1\right)>0$, and also $\operatorname{Pr}(D=1)>0$ and $\operatorname{Pr}(D=0)>0$, then we have the bounds

$$
\mathrm{LB}_{t} \leq \text { ATETS }_{t} \leq \mathrm{UB}_{t}
$$

where

$$
\begin{array}{r}
\mathrm{LB}_{t}=\operatorname{Pr}\left(Y_{t}=1 \mid \bar{Y}_{t-1}=0, D=1\right) \\
-\min \left\{1,1+\frac{\operatorname{Pr}\left(Y_{t}=1 \mid \bar{Y}_{t-1}=0, D=0\right) \operatorname{Pr}\left(\bar{Y}_{t-1}=0 \mid D=0\right)}{\operatorname{Pr}\left(\bar{Y}_{t-1}=0 \mid D=1\right)}\right. \\
\left.\quad-\frac{\min \left\{\operatorname{Pr}\left(\bar{Y}_{t-1}=0 \mid D=1\right), \operatorname{Pr}\left(\bar{Y}_{t-1}=0 \mid D=0\right)\right\}}{\operatorname{Pr}\left(\bar{Y}_{t-1}=0 \mid D=1\right)}\right\}, \\
\mathrm{UB}_{t}=\operatorname{Pr}\left(Y_{t}=1 \mid \bar{Y}_{t-1}=0, D=1\right) \\
-\max \left\{0, \frac{\left[\operatorname{Pr}\left(Y_{t}=1 \mid \bar{Y}_{t-1}=0, D=0\right)-1\right] \operatorname{Pr}\left(\bar{Y}_{t-1}=0 \mid D=0\right)}{\operatorname{Pr}\left(\bar{Y}_{t-1}=0 \mid D=1\right)}\right. \\
\left.+\frac{\min \left\{\operatorname{Pr}\left(\bar{Y}_{t-1}=0 \mid D=1\right), \operatorname{Pr}\left(\bar{Y}_{t-1}=0 \mid D=0\right)\right\}}{\operatorname{Pr}\left(\bar{Y}_{t-1}=0 \mid D=1\right)}\right\} .
\end{array}
$$

Proof. See Appendix A. 
Assumption 3 states that the treatment effect is either non-negative or non-positive for all $i$. Since in period 1 we can estimate the ATETS directly because there is no dynamic selection yet, the possibility that MTR holds with a non-positive effect, can be excluded if the ATETS in period 1 is non-negative. If we make the stronger assumption that the effect has the same sign for all $i$ and for all $t$ then a non-negative ATETS in period 1 excludes non-positive MTR in all periods. In that case the ATETS is non-negative in all time periods and this improves the lower bound on the ATETS, but has no effect on the upper bound that is between 0 and 1 . The lower bound on the ATETS if non-negative MTR holds is ${ }^{13}$ :

$$
\begin{aligned}
\mathrm{LB}_{t}= & \max \left\{0, \operatorname{Pr}\left(Y_{t}=1 \mid \bar{Y}_{t-1}=0, D=1\right)\right. \\
& \left.-\frac{\operatorname{Pr}\left(Y_{t}=1 \mid \bar{Y}_{t-1}=0, D=0\right) \operatorname{Pr}\left(\bar{Y}_{t-1}=0 \mid D=0\right)}{\operatorname{Pr}\left(\bar{Y}_{t-1}=0 \mid D=1\right)}\right\} .
\end{aligned}
$$

If MTR can change sign between periods we would require prior knowledge of the sign in each time period to improve on the bounds in Theorem 2 .

Theorem 3 (Bounds on ATETS Under PCO for $t$ Periods). Let Assumptions 1 and 5 hold. Let $t \in\{2,3,4, \ldots\}$. If $\operatorname{Pr}\left(\bar{Y}_{t-1}=0 \mid D=\right.$ 1) $=0$, then ATETS $_{t}$ is not defined.

If $\operatorname{Pr}\left(\bar{Y}_{t-1}=0 \mid D=1\right)>0$ and $\operatorname{Pr}\left(Y_{s}=0 \mid \bar{Y}_{s-1}=0, D=1\right)+\operatorname{Pr}\left(Y_{s}=0 \mid \bar{Y}_{s-1}=0, D=0\right)-1>0$ for all $s=1, \ldots, t-1$, and also $\operatorname{Pr}(D=1)>0$ and $\operatorname{Pr}(D=0)>0$, then we have the bounds

$$
\mathrm{LB}_{t} \leq \mathrm{ATETS}_{t} \leq \mathrm{UB}_{t},
$$

where

$$
\begin{aligned}
& \mathrm{LB}_{t}=\operatorname{Pr}\left(Y_{t}=1 \mid D=1, \bar{Y}_{t-1}=0\right)-1+\frac{1-\operatorname{Pr}\left(Y_{t}=1 \mid \bar{Y}_{t-1}=0, D=0\right)}{\operatorname{Pr}\left(\bar{Y}_{t-1}=0 \mid D=1\right)} \\
& \quad \times \prod_{s=1}^{t-1}\left[\operatorname{Pr}\left(Y_{s}=0 \mid \bar{Y}_{s-1}=0, D=1\right)+\operatorname{Pr}\left(Y_{s}=0 \mid \bar{Y}_{s-1}=0, D=0\right)-1\right], \\
& \mathrm{UB}_{t}=\operatorname{Pr}\left(Y_{t}=1 \mid D=1, \bar{Y}_{t-1}=0\right) \\
& -\max \left\{0, \frac{\left(\operatorname{Pr}\left(Y_{t}=1 \mid \bar{Y}_{t-1}=0, D=0\right)-1\right) \operatorname{Pr}\left(\bar{Y}_{t-1}=0 \mid D=0\right)}{\prod_{s=1}^{t-1}\left[\operatorname{Pr}\left(Y_{s}=0 \mid \bar{Y}_{s-1}=0, D=1\right)+\operatorname{Pr}\left(Y_{s}=0 \mid \bar{Y}_{s-1}=0, D=0\right)-1\right]}+1\right\} .
\end{aligned}
$$

If $\operatorname{Pr}\left(\bar{Y}_{t-1}=0 \mid D=1\right)>0$ and $\operatorname{Pr}\left(Y_{s}=0 \mid \bar{Y}_{s-1}=0, D=1\right)+\operatorname{Pr}\left(Y_{s}=0 \mid \bar{Y}_{s-1}=0, D=0\right)-1 \leq 0$ for some $s \leq t$, then we have the bounds

$$
\operatorname{Pr}\left(Y_{t}=1 \mid \bar{Y}_{t-1}=0, D=1\right)-1 \leq \operatorname{ATETS}_{t} \leq \operatorname{Pr}\left(Y_{t}=1 \mid \bar{Y}_{t-1}=0, D=1\right) .
$$

Proof. See Appendix A.

Theorem 4 (Bounds on ATETS Under MTR, CS and PCO for $t$ Periods). Let Assumptions 1 and 3-5 hold. Let $t \in\{2,3,4, \ldots\}$. If $\operatorname{Pr}\left(\bar{Y}_{t-1}=0 \mid D=1\right)=0$, then ATETS $_{t}$ is not defined.

If $\operatorname{Pr}\left(\bar{Y}_{t-1}=0 \mid D=1\right)>0$, and also $\operatorname{Pr}(D=1)>0$ and $\operatorname{Pr}(D=0)>0$, then we have the following bounds

$$
\mathrm{LB}_{t} \leq \mathrm{ATETS}_{t} \leq \mathrm{UB}_{t},
$$

where

$$
\begin{aligned}
\mathrm{LB}_{t}= & \operatorname{Pr}\left(Y_{t}=1 \mid D=1, \bar{Y}_{t-1}=0\right)-1+\frac{1-\operatorname{Pr}\left(Y_{t}=1 \mid \bar{Y}_{t-1}=0, D=0\right)}{\operatorname{Pr}\left(\bar{Y}_{t-1}=0 \mid D=1\right)} \\
& \times \min \left\{\operatorname{Pr}\left(\bar{Y}_{t-1}=0 \mid D=1\right), \operatorname{Pr}\left(\bar{Y}_{t-1}=0 \mid D=0\right)\right\}, \\
\mathrm{UB}_{t}= & \operatorname{Pr}\left(Y_{t}=1 \mid D=1, \bar{Y}_{t-1}=0\right) \\
& -\max \left\{0, \frac{\left(\operatorname{Pr}\left(Y_{t}=1 \mid \bar{Y}_{t-1}=0, D=0\right)-1\right) \operatorname{Pr}\left(\bar{Y}_{t-1}=0 \mid D=0\right)}{\min \left\{\operatorname{Pr}\left(\bar{Y}_{t-1}=0 \mid D=1\right), \operatorname{Pr}\left(\bar{Y}_{t-1}=0 \mid D=0\right)\right\}}+1\right\} .
\end{aligned}
$$

Proof. See Appendix A.

\footnotetext{
13 In the same way, if the ATETS in period 1 is non-positive, the possibility that MTR holds with a non-negative effect can be excluded, affecting the upper bound in an obvious way.
} 


\section{Inference}

Initially, for a given time period $t$, we consider inference on $\theta_{0}=$ ATETS $_{t}$ based on the identification result in Theorem 1. We assume that $\operatorname{Pr}\left(\bar{Y}_{t-1}=0 \mid D=1\right)>0$. The bounds in the theorem can then be expressed as

$$
\max \left(a_{1}, a_{2}\right)=: \ell \leq \theta_{0} \leq u:=\min \left(a_{3}, a_{4}\right),
$$

with

$$
\begin{aligned}
& a_{1}=a_{3}-1, \\
& a_{2}=a_{3}-\frac{1-\left[1-\operatorname{Pr}\left(Y_{t}=1 \mid \bar{Y}_{t-1}=0, D=0\right)\right] \operatorname{Pr}\left(\bar{Y}_{t-1}=0 \mid D=0\right)}{\operatorname{Pr}\left(\bar{Y}_{t-1}=0 \mid D=1\right)}, \\
& a_{3}=\operatorname{Pr}\left(Y_{t}=1 \mid \bar{Y}_{t-1}=0, D=1\right), \\
& a_{4}=a_{3}-1+\frac{1-\operatorname{Pr}\left(Y_{t}=1 \mid \bar{Y}_{t-1}=0, D=0\right) \operatorname{Pr}\left(\bar{Y}_{t-1}=0 \mid D=0\right)}{\operatorname{Pr}\left(\bar{Y}_{t-1}=0 \mid D=1\right)} .
\end{aligned}
$$

If we observe an iid sample $\left\{\left(Y_{i 1}, Y_{i 2}, \ldots, Y_{i t}, D_{i}\right), i \in 1, \ldots, n\right\}$, then the sample analog of $a=\left(a_{1}, a_{2}, a_{3}, a_{4}\right)^{\prime}$ can easily be constructed, for example

$$
\widehat{a}_{3}=\frac{\frac{1}{n} \sum_{i=1}^{n} \mathbb{1}\left(Y_{i t}=1, Y_{i 1}=0, Y_{i 2}=0, \ldots, Y_{i, t-1}=0, D_{i}=0\right)}{\frac{1}{n} \sum_{i=1}^{n} \mathbb{1}\left(Y_{i 1}=0, Y_{i 2}=0, \ldots, Y_{i, t-1}=0, D_{i}=0\right)}, \quad \widehat{a}_{1}=\widehat{a}_{3}-1,
$$

and analogously for $\widehat{a}_{2}$ and $\widehat{a}_{4}$. It is easy to show that as the sample size $n$ goes to infinity

$$
\sqrt{n}(\widehat{a}-a) \Rightarrow \mathcal{N}\left(0, \Sigma_{a}\right),
$$

and we can construct a consistent estimator $\widehat{\Sigma}_{a}$ of the $4 \times 4$ matrix $\Sigma_{a}$ (for example, we use bootstrapping to calculate $\widehat{\Sigma}_{a}$ in our application in Section 6). In the following we assume that $\Sigma_{a, k k}>0$ for all $k=1,2,3,4 .{ }^{14}$

The identification results in Theorem 2 for $\theta_{0}=$ ATETS $_{t}$ can also be expressed as max $\left(a_{1}, \min \left(a_{2}, a_{3}\right)\right) \leq \theta_{0} \leq$ $\min \left(a_{4}, \max \left(a_{5}, a_{6}\right)\right)$, with appropriate definition of $a=\left(a_{1}, a_{2}, a_{3}, a_{4}, a_{5}, a_{6}\right)^{\prime}$, whose estimator is again jointly normally distributed asymptotically, and the inference discussion below can be easily generalized to this case. Similarly with Theorems 3 and 4 .

\subsection{Connection to the moment inequality literature}

The inference problem for $\theta_{0}$ that is summarized by (10) and (11) is asymptotically equivalent to an inference problem on a finite number of moment inequalities that is well-studied in the literature, for example in Chernozhukov et al. (2007), Romano and Shaikh (2008), Rosen (2008), Andrews and Guggenberger (2009), Andrews and Soares (2010), and Andrews and Barwick (2012). To make this connection explicit we define

$$
m(\theta):=\left(\begin{array}{c}
\Sigma_{a, 11}^{-1 / 2}\left(a_{1}-\theta\right) \\
\Sigma_{a, 22}^{-1 / 2}\left(a_{2}-\theta\right) \\
\Sigma_{a, 33}^{-1 / 2}\left(\theta-a_{3}\right) \\
\Sigma_{a, 44}^{-1 / 2}\left(\theta-a_{4}\right)
\end{array}\right), \quad \widehat{m}(\theta):=\left(\begin{array}{l}
\widehat{\Sigma}_{a, 11}^{-1 / 2}\left(\widehat{a}_{1}-\theta\right) \\
\widehat{\Sigma}_{a, 22}^{-1 / 2}\left(\widehat{a}_{2}-\theta\right) \\
\widehat{\Sigma}_{a, 33}^{-1 / 2}\left(\theta-\widehat{a}_{3}\right) \\
\widehat{\Sigma}_{a, 44}^{-1 / 2}\left(\theta-\widehat{a}_{4}\right)
\end{array}\right) .
$$

The bounds (10) can then equivalently be expressed as $m\left(\theta_{0}\right) \leq 0$, which is analogous to imposing four moment inequalities. ${ }^{15}$ For convenience we have normalized $m(\theta)$ such that each component of $\sqrt{n}[\widehat{m}(\theta)-m(\theta)]$ has asymptotic variance equal to one. Using (11) we obtain $\sqrt{n}[\widehat{m}(\theta)-m(\theta)] \Rightarrow \mathcal{N}\left(0, \Sigma_{m}\right)$, where $\Sigma_{m}=A \Sigma_{a} A$, with $A=$ $\operatorname{diag}\left(\Sigma_{a, 11}^{-1 / 2}, \Sigma_{a, 22}^{-1 / 2},-\Sigma_{a, 33}^{-1 / 2},-\Sigma_{a, 44}^{-1 / 2}\right)$. An estimator $\widehat{\Sigma}_{m}$ can be constructed analogously.

All the papers on moment inequalities cited above start from choosing an objective function (or criterion function, or test statistics), whose sample version we denote by $\widehat{Q}(\theta)$, and then construct a confidence set for $\theta_{0}$ as

$$
\widehat{\Theta}\left(C_{1-\alpha}\right)=\left\{\theta \in \mathbb{R}: n \widehat{Q}(\theta) \leq C_{1-\alpha}\right\},
$$

\footnotetext{
14 Since $\widehat{a}_{1}$ and $\widehat{a}_{3}$ are perfectly correlated we have $\Sigma_{a} v=0$ for the vector $v=(1,-1,0,0)^{\prime}$, implying that $\operatorname{rank}\left(\Sigma_{a}\right) \leq 3$, but this rank deficiency turns out not to be important for our purposes.

$15 m(\theta)$ is not actually a moment function, but has a slightly more complicated structure (e.g. $a_{3}$ is a conditional probability that can be expressed as the ratio between two moments). This, however, does not matter for the asymptotic analysis since the estimator $\widehat{m}(\theta)$ has the same first order asymptotic properties as it would have in the moment inequality case. We can therefore fully draw on the insights of the existing literature.
} 
where $C_{1-\alpha} \geq 0$ is a critical value that is chosen such that confidence $1-\alpha$ is achieved asymptotically, i.e. $\lim _{n \rightarrow \infty} \operatorname{Pr}\left(\theta_{0} \in\right.$ $\left.\widehat{\Theta}\left(C_{1-\alpha}\right)\right) \geq 1-\alpha .{ }^{16}$ Various objective functions have been considered in the literature. For example, the objective function considered in Chernozhukov et al. (2007) reads in our notation $\widehat{Q}(\theta)=\left\|[\widehat{m}(\theta)]_{+}\right\|^{2}$, where $\|$.$\| refers to the Euclidian norm,$ and $[\widehat{m}(\theta)]_{+}:=\max (0, \widehat{m}(\theta))$, applied componentwise to the vector $\widehat{m}(\theta)$.

\subsection{Construction of confidence intervals}

Our specific inference problem is easier than the general inference problem for moment inequalities, because in our case the parameter $\theta_{0}$ is just a scalar, and the total number of inequalities is relatively small. Our goal in the following is therefore to outline a concrete method of how to construct a confidence interval in that special case.

We choose the objective function $\widehat{Q}(\theta)=\left\|[\widehat{m}(\theta)]_{+}\right\|_{\infty}^{2}$, where $\|\cdot\|_{\infty}$ is the infinity norm, ${ }^{17}$ i.e. we have $\widehat{Q}(\theta)=$ $\max \left\{0, \widehat{m}_{1}(\theta), \widehat{m}_{2}(\theta), \widehat{m}_{3}(\theta), \widehat{m}_{4}(\theta)\right\}^{2}$. This objective function is convenient for our purposes, because the confidence set defined above then takes the intuitive form

$$
\begin{aligned}
& \widehat{\Theta}\left(C_{1-\alpha}\right) \\
& =\left[\max \left(\widehat{a}_{1}-\frac{c_{1-\alpha} \widehat{\Sigma}_{a, 11}^{1 / 2}}{\sqrt{n}}, \widehat{a}_{2}-\frac{c_{1-\alpha} \widehat{\Sigma}_{a, 22}^{1 / 2}}{\sqrt{n}}\right), \min \left(\widehat{a}_{3}+\frac{c_{1-\alpha} \widehat{\Sigma}_{a, 33}^{1 / 2}}{\sqrt{n}}, \widehat{a}_{4}+\frac{c_{1-\alpha} \widehat{\Sigma}_{a, 44}^{1 / 2}}{\sqrt{n}}\right)\right],
\end{aligned}
$$

where $c_{1-\alpha}:=\sqrt{C_{1-\alpha}}$. This confidence interval can be constructed very easily.

\section{Most robust critical value}

The critical value $c_{1-\alpha}$ still needs to be chosen. The problem with choosing the critical value in moment inequality problems is that this choice depends on the unknown slackness vector $m\left(\theta_{0}\right)$, which indicates whether each inequality $m_{k}\left(\theta_{0}\right) \leq 0$ is binding, close to binding, or far from binding. It is known, however, that the largest ("worst case") critical value needs to be chosen if $m\left(\theta_{0}\right)=0$, i.e. if all moment inequalities are binding at the true parameter. To find this critical value one can use the fact that in this worst case $n \widehat{Q}(\theta)$ is asymptotically distributed as $\left\|[Z]_{+}\right\|_{\infty}^{2}$, where $Z \sim \mathcal{N}\left(0, \Sigma_{m}\right)$ is a random four vector. Using the estimator $\widehat{\Sigma}_{m}$ one can simulate this distribution. However, it can easily be shown that the $1-\alpha$ quantile of $\left\|[Z]_{+}\right\|_{\infty}$ is always smaller or equal to the following conservative critical value

$$
c_{1-\alpha}=\Phi^{-1}\left(1-\frac{\alpha}{4}\right)
$$

where $\Phi^{-1}$ is the quantile function (the inverse cdf) of the standard normal distribution. The factor $1 / 4$ that appears here reflects the fact that we have four moment inequalities. Combining Eqs. (13) and (14) provides a confidence interval that is uniformly valid, i.e. whose asymptotic size is bounded by $\alpha$, independent of what the true values of $a_{1}, a_{2}, a_{3}$ and $a_{4}$ are.

\section{Critical value for the case $\ell \ll u$}

The critical values based on the "worst case" where all inequalities are binding $\left(m\left(\theta_{0}\right)=0\right)$ can be very conservative if one or multiple inequalities are far from binding $\left(m_{k}\left(\theta_{0}\right) \ll 0\right) .{ }^{18}$ Furthermore, for the inference on $\theta_{0}=$ ATETS ${ }_{t}$ based on Theorem 1, with $a$ 's as given above, it can easily be shown that if $\operatorname{Pr}\left(\bar{Y}_{t-1}=0 \mid D=1\right)>0$ and $\operatorname{Pr}\left(\bar{Y}_{t-1}=0 \mid D=0\right)<1$, then we have $\max \left(a_{1}, a_{2}\right)=: \ell<u:=\min \left(a_{3}, a_{4}\right)$, implying that $m\left(\theta_{0}\right)=0$ is impossible. However, what matters for the coverage rate of the confidence interval for a finite sample is not whether $\ell<u$, but whether the difference $u-\ell$ is large relative to the standard deviations $\Sigma_{a, k k}^{1 / 2}$ of the $\widehat{a}_{k}, k=1,2,3,4$. This is what we mean by $\ell \ll u$ in the subsection title above.

To formalize this one can consider a pretest of the hypothesis $H_{0}: \ell=u$, against the alternative $H_{a}: \ell<u$, with pretest size $\alpha_{n}^{\text {pre }}$ chosen to be very small, e.g. $\alpha_{n}^{\text {pre }}=0.001 \ll \alpha{ }^{19}$ If the pretest is not rejected, then the critical value (14) should be chosen. If the pretest is rejected, then the two problems of choosing a suitable lower and upper bound for the confidence interval $\widehat{\Theta}$ completely decouple, because with high confidence we know that for any $\theta$ only one of those bounds can be binding at the same time, implying that at most two of the moment inequalities $m\left(\theta_{0}\right) \leq 0$ can be binding. In this latter case we can therefore choose the less conservative critical value

$$
c_{1-\alpha}=\Phi^{-1}\left(1-\frac{\alpha}{2}\right)
$$

when computing the confidence interval (13).

\footnotetext{
16 As discussed in e.g. Andrews and Soares (2010), it is important that the coverage probability is asymptotically bounded by $1-\alpha$ uniformly over $\theta_{0}$ and over the distribution of the observables. We have only formulated the pointwise condition here to keep the presentation simple.

17 This is special case of the "test function" $S_{3}(m, \Sigma)$ introduced in equation (3.6) of Andrews and Soares (2010), with $p_{1}=1$ and $v=0$ in their notation.

18 In addition, the formula (14) only provides an upper bound for the optimal critical value at $m\left(\theta_{0}\right)=0$, but this second issue is often not very severe. For example, for $\alpha=0.05$ and $\Sigma_{m}=\mathbb{I}_{4}$ one finds by simulation that the 0.95 quantile of $\left\|[Z]_{+}\right\|_{\infty}$, with $Z \sim \mathcal{N}\left(0, \Sigma_{m}\right)$, is $c_{0.95}=2.234$, while the much easier to computer conservative critical value in (14) is $\Phi^{-1}(0.9875)=2.241$.

19 Theoretically one can assume $\alpha_{n}^{\text {pre }} \rightarrow 0$ as $n \rightarrow \infty$ to avoid asymptotic size distortions due to the pretest.
} 
Critical value for the case $a_{1} \ll a_{2} \ll u$

Analogous to the discussion of (14), the critical value (15) is again potentially conservative because it is based on the case where two of the inequalities $m\left(\theta_{0}\right) \leq 0$ (for either the lower or the upper bound, respectively) are jointly binding. ${ }^{20}$ For example, if we find that $a_{1} \ll a_{2} \ll u$ (by which we again mean that the null hypotheses $H_{0}: a_{1}=a_{2}$, vs. $H_{a}: a_{1}<a_{2}$, and $H_{0}: a_{2}=u$, vs. $H_{a}: a_{2}<u$, are rejected with very high confidence), then a natural confidence interval to report is

$$
\widehat{\Theta}=\left[\widehat{a}_{2}-\frac{\Phi^{-1}(1-\alpha) \widehat{\Sigma}_{a, 22}^{1 / 2}}{\sqrt{n}}, \min \left(\widehat{a}_{3}+\frac{\Phi^{-1}\left(1-\frac{\alpha}{2}\right) \widehat{\Sigma}_{a, 33}^{1 / 2}}{\sqrt{n}}, \widehat{a}_{4}+\frac{\Phi^{-1}\left(1-\frac{\alpha}{2}\right) \widehat{\Sigma}_{a, 44}^{1 / 2}}{\sqrt{n}}\right)\right] .
$$

Note that the lower bound of $\widehat{\Theta}$ now corresponds to inverting a standard one-sided t-test. Analogous confidence intervals can obviously be constructed in other cases, e.g. $\ell \ll a_{3} \ll a_{4}$ or $a_{2} \ll a_{1} \ll a_{4} \ll a_{3}$, etc.

The different critical values and corresponding confidence intervals discussed above correspond to cases where different subsets of the inequalities $m\left(\theta_{0}\right) \leq 0$ can be simultaneously binding, i.e. to a moment selection problem. A much more general discussion of moment selection is given e.g. in Andrews and Soares (2010). Different confidence intervals than those discussed here, e.g. based on different objective functions $\widehat{Q}(\theta)$, can of course also be considered.

It should be noted that pretesting is not required if we use the approach in Hahn and Ridder (2014) who obtain a confidence interval by inverting the Likelihood Ratio test for the composite null and composite alternative test. Their current results do not cover the case considered here and we did not attempt the non-trivial extension to the case considered here.

\section{Application to the Illinois bonus experiment}

\subsection{The re-employment bonus experiment}

In 1984, the Illinois Department of Employment Security conducted a randomized social experiment. ${ }^{21}$ The goal of the experiment was to explore, whether re-employment bonuses paid to Unemployment Insurance (UI) beneficiaries (treatment 1) or their employers (treatment 2) reduced the length of unemployment spells.

Both treatments consisted of a $\$ 500$ re-employment bonus, which was about four times the average weekly unemployment insurance benefit. In the experiment, newly unemployed UI claimants were randomly divided into three groups:

1. The Claimant Bonus Group. The members of this group were instructed that they would qualify for a cash bonus of $\$ 500$ if they found a job (of at least $30 \mathrm{~h}$ ) within 11 weeks and, if they held that job for at least 4 months. A total of 4186 individuals were selected for this group, and 3527 (84\%) agreed to participate.

2. The Employer Bonus Group. The members of this group were told that their next employer would qualify for a cash bonus of $\$ 500$ if they, the claimants, found a job (of at least $30 \mathrm{~h}$ ) within 11 weeks and, if they held that job for at least four months. A total of 3963 were selected for this group and 2586 (65\%) agreed to participate.

3. The Control Group, i.e. all claimants not assigned to one of the treatment groups. This group consisted of 3952 individuals. The individuals assigned to the control group were excluded from participation in the experiment. In fact, they did not know that the experiment took place.

The descriptive statistics in Table 2 in Woodbury and Spiegelman (1987) confirm that the randomization resulted in three similar groups.

\subsection{Results of previous studies}

Woodbury and Spiegelman (1987) concluded from a direct comparison of the control group and the two treatment groups that the claimant bonus group had a significantly shorter average unemployment duration. The average unemployment duration was also shorter for the employer bonus group, but the difference was not significantly different from zero. In Illinois UI benefits end after 26 weeks and since administrative data were used, all unemployment durations are censored at 26 weeks. Woodbury and Spiegelman ignore the censoring and take as outcome variable the number of weeks of insured unemployment.

Meyer (1996) analyzed the same data but focused on the treatment effects on conditional transition probabilities which allows him to properly account for censoring. Meyer focuses on the conditional transitions rates because both labor supply and search theory imply specific dynamic treatment effects. The bonus is only given to an unemployed individual if (s)he finds a job within 11 weeks and retains it for four months. The cash bonus is the same for all unemployed. Theory predicts that (i) the transition rate during the eligibility period (first 11 weeks) will be higher in the two treatment groups compared with the control group, and (ii) that the transition rate in the treatment groups will rise just before the end of the eligibility period, as the unemployed run out of time to collect the bonus.

To test these predictions, Meyer (1996) estimates a proportional hazard (PH) model with a flexible specification of the baseline hazard. He uses the treatment indicator as an explanatory variable. Since there was partial compliance with

\footnotetext{
20 It is also conservative, because the information in the correlation matrix $\Sigma_{m}$ is not used to construct (15). It corresponds to the most extreme case where both lower bound estimators $\widehat{a}_{1}$ and $\widehat{a}_{2}$ (or both upper bound estimators $\widehat{a}_{3}$ and $\widehat{a}_{4}$ ) are perfectly negatively correlated.

21 The population consisted of those who filled for UI between July 29, 1984 and November 17, 1984. A complete description of the experiment and a summary of its results can be found in Woodbury and Spiegelman (1987).
} 
treatment his estimator can be interpreted as a intention to treat (ITT) estimator. ${ }^{22}$ In his analysis Meyer controls for age, the logarithm of base period earnings, ethnicity , gender and the logarithm of the size of the UI benefits. He finds a significantly positive effect of the claimant bonus and a positive but insignificant effect of the employer bonus. A more detailed analysis of the effects for the claimant group reveals a positive effect on the transition rate during the first 11 weeks in unemployment, an increased effect during week 9 and 10, and no significant effect on the transition rate after week 11 as predicted by labor supply and search theory.

\subsection{Estimates of bounds}

In his study Meyer (1996) relies on the proportionality of the hazard rate to investigate his hypotheses. We now ask what can be said if the assumptions of the MPH model do not hold, that is what can be identified if we rely solely on random assignment and the additional assumptions. As in Meyer (1996) we consider the ITT effect, that is, we do not correct for partial compliance. We divide the 24 month observation period into 12 subperiods: week $1-2$, week 3-4, .., week 23-24. The reason for this is that there is a pronounced even-odd week effect in the data, with higher transition rate during odd weeks. With these subperiods the predictions we wish to test are: (i) a positive treatment effect during periods $1-5$, i.e.

$$
\text { ATETS }_{t}>0, t=1, \ldots, 5,
$$

(ii) no effect after the bonus offer has expired in periods $6-12$, i.e.

$$
\text { ATETS }_{t}=0, t=6, \ldots, 12,
$$

and (iii) a larger effect of the bonus offer at the end of the eligibility period in period 5, i.e.

$$
\text { ATETS }_{5}>\text { ATETS }_{4} \text {. }
$$

Note that in this experiment the treatment assignment is in period 1 , so that in ATETS $_{t}$ the superscripts 1 and 0 are $t$ vectors with components equal to 1 and 0 .

We report both the bounds that are obtained by simply replacing the population moments with their sample analogs, as well as the confidence intervals based on the approach described in Section $5 .^{23}$ Table 2 presents the upper and the lower bound and the confidence interval on ATETS $_{t}$ for the claimant group assuming only random assignment. We find that the instantaneous treatment effect on the transition probability (week 1-2) is point identified and indicates a positive effect of the re-employment bonus. The transition probability is about 2 percentage points higher in the treatment group compared to the control group. This estimate is statistically significant. From week 3-4 and onwards the bounds are quite wide. In fact, without further assumptions we cannot rule out that the bonus actually has a negative impact on the conditional transition probability after week 3 . However, the bounds are nevertheless informative on the average treatment effect in all time periods.

Table 2 also shows that the confidence intervals are marginally wider than the actual bounds. That is the uncertainty arising from the dynamic selection is far greater than the uncertainty due to sampling variation.

Next, Table 2 presents bounds under the additional assumptions in Section 4. As expected, if we impose additional assumptions the bounds are considerably narrower. Under MTR and CS we can rule out very large negative and very large positive dynamic treatment effects. Imposing MTR, CS as well as PCO further tightens the bounds. If these assumptions hold simultaneously we can, if we disregard sampling variation, rule out that the bonus offer has a negative effect on the transition rate out of unemployment up to week 20 . This conclusions changes slightly when sampling variation is taken into account.

Let us return to the three hypotheses suggested by labor supply and search theory, and consider our most restrictive bounds under MTR, CS and PCO. We find that there is a positive effect of the bonus offer on the conditional transition rate up to week 11 . This confirms the first hypothesis. The upper bound increases in time period 5 (weeks 9-10), but the lower bound does not increase enough, so that both an increase and no change (and even a small decrease) in the transition probability out of unemployment are consistent with the data. Now consider the third hypothesis that there is no effect on the transition rate after week 11. Again the bounds do not rule out that there is a positive effect on the conditional transition probability after week 11. These results illustrate that the evidence for the second and third hypotheses presented by a number of authors rely on the imposed structure, e.g. proportionality of the hazard or the restrictions implied by a particular discrete-time duration model.

We next examine heterogeneous effects. To this end we split our sample by gender, race and pre-unemployment income and estimate our bounds for each subgroup. We provide results for bounds without additional assumptions and bounds under MTR, CS and PCO. The other bounds are available upon request. If we focus on the bounds under MTR, CS and PCO,

\footnotetext{
22 The partial compliance is addressed in detail by Bijwaard and Ridder (2005). They introduce a new method to handle the selective compliance in the treatment group. If there is full compliance in the control group, their two-stage linear rank estimator is able to handle the selective compliance in the treatment group even for censored durations. In order to achieve this they assume a MPH structure for the transition rate. Their estimates indicate that the ITT estimates by Meyer (1996) underestimate the true treatment effect.

23 The covariance matrix $\Sigma_{a}$ is estimated using the bootstrap with 399 replications. Constructing confidence intervals furthermore requires moment selection, e.g. for the bounds under just random assignment we find that with very high confidence only one inequality is binding for the lower as well as the upper bound. Details are available from the authors upon request.
} 
Table 2

Bounds on ATETS $^{1,0}$ for the Illinois job-bonus experiment.

\begin{tabular}{|c|c|c|c|c|c|c|c|c|}
\hline & \multicolumn{4}{|c|}{ No assumption bounds $[\mathrm{A}]$} & \multicolumn{4}{|c|}{ MTR + CS [B] } \\
\hline & $\begin{array}{l}\text { Lower-CI } \\
\text { (1) }\end{array}$ & $\begin{array}{l}\text { LB } \\
(2)\end{array}$ & $\begin{array}{l}\text { UB } \\
\text { (3) }\end{array}$ & $\begin{array}{l}\text { Upper-CI } \\
(4)\end{array}$ & $\begin{array}{l}\text { Lower-CI } \\
\text { (5) }\end{array}$ & $\begin{array}{l}\text { LB } \\
(6)\end{array}$ & $\begin{array}{l}\text { UB } \\
(7)\end{array}$ & $\begin{array}{l}\text { Upper-CI } \\
\text { (8) }\end{array}$ \\
\hline \multicolumn{9}{|l|}{ Week } \\
\hline $1-2$ & 0.012 & 0.023 & 0.023 & 0.034 & 0.012 & 0.023 & 0.023 & 0.034 \\
\hline $3-4$ & -0.145 & -0.137 & 0.094 & 0.102 & 0.000 & 0.011 & 0.038 & 0.050 \\
\hline $5-6$ & -0.259 & -0.251 & 0.074 & 0.082 & -0.007 & 0.004 & 0.046 & 0.056 \\
\hline $7-8$ & -0.346 & -0.339 & 0.078 & 0.086 & 0.004 & 0.013 & 0.063 & 0.073 \\
\hline $9-10$ & -0.452 & -0.444 & 0.069 & 0.077 & 0.000 & 0.008 & 0.069 & 0.079 \\
\hline $11-12$ & -0.552 & -0.544 & 0.062 & 0.070 & 0.000 & 0.008 & 0.062 & 0.072 \\
\hline $13-14$ & -0.655 & -0.648 & 0.056 & 0.064 & -0.010 & -0.002 & 0.056 & 0.064 \\
\hline $15-16$ & -0.750 & -0.743 & 0.051 & 0.058 & -0.004 & 0.003 & 0.051 & 0.058 \\
\hline $17-18$ & -0.844 & -0.836 & 0.049 & 0.057 & -0.007 & 0.000 & 0.049 & 0.057 \\
\hline $19-20$ & -0.943 & -0.936 & 0.049 & 0.057 & -0.011 & -0.004 & 0.049 & 0.056 \\
\hline $21-22$ & -0.994 & -0.953 & 0.047 & 0.056 & -0.028 & -0.021 & 0.047 & 0.055 \\
\hline \multirow[t]{3}{*}{$23-24$} & -0.989 & -0.944 & 0.056 & 0.064 & -0.011 & -0.002 & 0.056 & 0.064 \\
\hline & \multicolumn{4}{|l|}{$\mathrm{PCO}[\mathrm{C}]$} & \multicolumn{4}{|c|}{$\mathrm{MTR}+\mathrm{CS}+\mathrm{PCO}[\mathrm{D}]$} \\
\hline & $\begin{array}{l}\text { Lower-CI } \\
\text { (1) }\end{array}$ & $\begin{array}{l}\text { LB } \\
(2)\end{array}$ & $\begin{array}{l}\text { UB } \\
(3)\end{array}$ & $\begin{array}{l}\text { Upper-CI } \\
\text { (4) }\end{array}$ & $\begin{array}{l}\text { Lower-CI } \\
\text { (5) }\end{array}$ & $\begin{array}{l}\text { LB } \\
(6)\end{array}$ & $\begin{array}{l}\text { UB } \\
(7)\end{array}$ & $\begin{array}{l}\text { Upper-CI } \\
\text { (8) }\end{array}$ \\
\hline \multicolumn{9}{|l|}{ Week } \\
\hline $1-2$ & 0.012 & 0.023 & 0.023 & 0.034 & 0.012 & 0.023 & 0.023 & 0.034 \\
\hline $3-4$ & -0.131 & -0.123 & 0.094 & 0.102 & 0.002 & 0.014 & 0.038 & 0.049 \\
\hline $5-6$ & -0.209 & -0.202 & 0.074 & 0.082 & -0.004 & 0.007 & 0.046 & 0.055 \\
\hline $7-8$ & -0.256 & -0.247 & 0.078 & 0.087 & 0.008 & 0.016 & 0.063 & 0.072 \\
\hline $9-10$ & -0.306 & -0.299 & 0.069 & 0.077 & 0.004 & 0.012 & 0.069 & 0.078 \\
\hline $11-12$ & -0.348 & -0.340 & 0.062 & 0.070 & 0.004 & 0.012 & 0.062 & 0.071 \\
\hline $13-14$ & -0.388 & -0.379 & 0.056 & 0.064 & -0.004 & 0.003 & 0.056 & 0.064 \\
\hline $15-16$ & -0.419 & -0.411 & 0.051 & 0.058 & 0.000 & 0.007 & 0.051 & 0.059 \\
\hline $17-18$ & -0.445 & -0.438 & 0.049 & 0.057 & -0.003 & 0.005 & 0.049 & 0.058 \\
\hline $19-20$ & -0.472 & -0.464 & 0.049 & 0.057 & -0.006 & 0.001 & 0.049 & 0.057 \\
\hline $21-22$ & -0.504 & -0.496 & 0.047 & 0.063 & -0.022 & -0.014 & 0.047 & 0.055 \\
\hline $23-24$ & -0.523 & -0.513 & 0.056 & 0.073 & -0.006 & 0.003 & 0.056 & 0.065 \\
\hline
\end{tabular}

Notes: CI is $95 \%$ confidence intervals. Variances and covariances used to obtain the CI are estimated using bootstrap (399 replications).

Table 3 indicates several interesting differences between males and females. For males we find significant effects in the beginning of the unemployment spell (weeks 1-2) and shortly before the bonus expires (weeks 7-10). For females on the other hand we only find significant effects in weeks $1-4$, but no effects in weeks $5-11$. This indicates that females quickly responds to the bonus offer, whereas a large part of the effects for males occur shortly before the end of the subsidy. Table B1 in Appendix B also reveals some differences between blacks and non-blacks. For both groups we find significant effects during the first 11 weeks of unemployment, but for non-blacks the bonus offer also increases the transition rates after the bonus offers has expired (e.g. during weeks 15-16). Finally, Table B2 in Appendix B reveals no significant differences between how workers with low and high income react to the bonus offer.

\section{Conclusions}

In this paper, we have derived bounds on treatment effects on conditional transition probabilities under (sequential) randomization. The partial identification problem arises since random assignment only ensures comparability of the treatment and control groups at the time of randomization. In the literature this problem is often refereed to as the dynamic selection problem. For that reason only instantaneous or short-run effects are point identified, whereas dynamic or long-run effects in general are not point identified. Our weakest bounds impose no assumptions beyond (sequential) random assignment, so that they are not sensitive to arbitrary functional form assumptions, require no additional covariates and allow arbitrary heterogeneous treatment effects as well as arbitrary unobserved heterogeneity. These non-parametric bounds offer an alternative to semi-parametric methods. They tend to be wide and therefore we have also derived more informative bounds under additional assumptions that often hold in semi-parametric reduced form and structural models.

An analysis of data from the Illinois re-employment bonus experiment shows that our bounds are informative about average treatment effects. It also demonstrates that previous results on the evolution of the average treatment effect require assumptions such as the proportionality of the hazard rate or those embodied in a particular (semi-)parametric discrete-time hazard model.

\section{Acknowledgments}

We are grateful for helpful suggestions from the editor Han Hong, an associate editor, two anonymous referees, John Ham, Per Johansson, Adam Rosen, Michael Svarer, Gerard van den Berg and seminar participants at IFAU-Uppsala, Uppsala 
Table 3

Bounds on ATETS $^{1,0}$ for the Illinois job-bonus experiment. Heterogeneous effects for males and females.

\begin{tabular}{|c|c|c|c|c|c|c|c|c|}
\hline & \multicolumn{8}{|c|}{ Panel A: Males } \\
\hline & \multicolumn{4}{|c|}{ No assumption bounds } & \multicolumn{4}{|c|}{$\mathrm{MTR}+\mathrm{CS}+\mathrm{PCO}$} \\
\hline & $\begin{array}{l}\text { Lower-CI } \\
\text { (1) }\end{array}$ & $\begin{array}{l}\text { LB } \\
(2)\end{array}$ & $\begin{array}{l}\text { UB } \\
(3)\end{array}$ & $\begin{array}{l}\text { Upper-CI } \\
(4)\end{array}$ & $\begin{array}{l}\text { Lower-CI } \\
\text { (1) }\end{array}$ & $\begin{array}{l}\text { LB } \\
(2)\end{array}$ & $\begin{array}{l}\text { UB } \\
(3)\end{array}$ & $\begin{array}{l}\text { Upper-CI } \\
\text { (4) }\end{array}$ \\
\hline \multicolumn{9}{|l|}{ Week } \\
\hline $1-2$ & -0.004 & 0.016 & 0.016 & 0.037 & 0.002 & 0.016 & 0.016 & 0.030 \\
\hline $3-4$ & -0.152 & -0.141 & 0.094 & 0.105 & -0.004 & 0.009 & 0.026 & 0.039 \\
\hline $5-6$ & -0.269 & -0.259 & 0.075 & 0.084 & -0.010 & 0.003 & 0.030 & 0.043 \\
\hline $7-8$ & -0.349 & -0.338 & 0.085 & 0.096 & 0.009 & 0.024 & 0.054 & 0.069 \\
\hline $9-10$ & -0.464 & -0.453 & 0.076 & 0.087 & 0.000 & 0.014 & 0.070 & 0.084 \\
\hline $11-12$ & -0.573 & -0.562 & 0.069 & 0.080 & 0.005 & 0.015 & 0.069 & 0.081 \\
\hline $13-14$ & -0.688 & -0.676 & 0.065 & 0.076 & -0.004 & 0.006 & 0.065 & 0.077 \\
\hline $15-16$ & -0.793 & -0.782 & 0.054 & 0.064 & 0.004 & 0.014 & 0.054 & 0.064 \\
\hline $17-18$ & -0.899 & -0.887 & 0.056 & 0.067 & -0.008 & 0.003 & 0.056 & 0.066 \\
\hline $19-20$ & -0.994 & -0.941 & 0.059 & 0.071 & -0.004 & 0.008 & 0.059 & 0.071 \\
\hline $21-22$ & -1.006 & -0.948 & 0.052 & 0.063 & -0.028 & -0.017 & 0.052 & 0.066 \\
\hline \multirow[t]{5}{*}{$23-24$} & -1.006 & -0.941 & 0.059 & 0.071 & -0.010 & 0.002 & 0.059 & 0.074 \\
\hline & \multicolumn{4}{|l|}{$\mathrm{PCO}[\mathrm{C}]$} & \multicolumn{4}{|c|}{$\mathrm{MTR}+\mathrm{CS}+\mathrm{PCO}[\mathrm{D}]$} \\
\hline & \multicolumn{8}{|c|}{ Panel B: Females } \\
\hline & \multicolumn{4}{|c|}{ No assumption bounds } & \multicolumn{4}{|c|}{$\mathrm{MTR}+\mathrm{CS}+\mathrm{PCO}$} \\
\hline & $(1)$ & $(2)$ & $(3)$ & $(4)$ & $(1)$ & $(2)$ & (3) & $(4)$ \\
\hline \multicolumn{9}{|l|}{ Week } \\
\hline $1-2$ & 0.008 & 0.031 & 0.031 & 0.054 & 0.014 & 0.031 & 0.031 & 0.047 \\
\hline $3-4$ & -0.143 & -0.131 & 0.093 & 0.105 & 0.003 & 0.019 & 0.053 & 0.069 \\
\hline $5-6$ & -0.251 & -0.239 & 0.074 & 0.085 & 0.000 & 0.012 & 0.066 & 0.080 \\
\hline $7-8$ & -0.348 & -0.337 & 0.068 & 0.079 & -0.006 & 0.005 & 0.068 & 0.082 \\
\hline $9-10$ & -0.441 & -0.430 & 0.060 & 0.071 & -0.003 & 0.009 & 0.060 & 0.073 \\
\hline $11-12$ & -0.528 & -0.517 & 0.053 & 0.064 & -0.002 & 0.008 & 0.053 & 0.066 \\
\hline $13-14$ & -0.616 & -0.606 & 0.045 & 0.055 & -0.011 & 0.000 & 0.045 & 0.055 \\
\hline $15-16$ & -0.698 & -0.686 & 0.046 & 0.057 & -0.012 & 0.000 & 0.046 & 0.059 \\
\hline $17-18$ & -0.775 & -0.764 & 0.041 & 0.052 & -0.008 & 0.007 & 0.041 & 0.055 \\
\hline $19-20$ & -0.861 & -0.851 & 0.036 & 0.047 & -0.016 & -0.006 & 0.036 & 0.047 \\
\hline $21-22$ & -0.949 & -0.936 & 0.041 & 0.054 & -0.022 & -0.011 & 0.041 & 0.055 \\
\hline $23-24$ & -1.020 & -0.948 & 0.052 & 0.066 & -0.009 & 0.004 & 0.052 & 0.068 \\
\hline
\end{tabular}

Notes: $\mathrm{CI}$ is $95 \%$ confidence intervals. Variances and covariances used to obtain the CI are estimated using bootstrap (399 replications).

University, University of Aarhus and University of Mannheim. We acknowledge support from the Jan Wallander and Tom Hedelius Foundation, NSF grants SES 0819612 and 0819638, from the ESRC Centre for Microdata Methods and Practice grant RES-589-28-0001, and from the European Research Council (ERC) grant ERC-2014-CoG-646917-ROMIA.

\section{Appendix A. Proofs}

\section{Proof of Theorem 1.}

We use the following notation for the distribution of the potential outcomes. For $d=0,1$

$$
\begin{aligned}
p_{t}^{d}(1 \mid 0,0) & =: \operatorname{Pr}\left(Y_{t}^{d}=1 \mid \bar{Y}_{t-1}^{1}=0, \bar{Y}_{t-1}^{0}=0\right), \\
p_{t}^{d}(1 \mid 0, \neq 0) & =: \operatorname{Pr}\left(Y_{t}^{d}=1 \mid \bar{Y}_{t-1}^{1}=0, \bar{Y}_{t-1}^{0} \neq 0\right), \\
p_{t}^{d}(1 \mid \neq 0,0) & =: \operatorname{Pr}\left(Y_{t}^{d}=1 \mid \bar{Y}_{t-1}^{1} \neq 0, \bar{Y}_{t-1}^{0}=0\right),
\end{aligned}
$$

and for the joint distribution of $\bar{Y}_{t-1}^{1}, \bar{Y}_{t-1}^{0}$

$$
\begin{aligned}
p_{t-1}(0,0) & =: \operatorname{Pr}\left(\bar{Y}_{t-1}^{1}=0, \bar{Y}_{t-1}^{0}=0\right), \\
p_{t-1}(0, \neq 0) & =: \operatorname{Pr}\left(\bar{Y}_{t-1}^{1}=0, \bar{Y}_{t-1}^{0} \neq 0\right), \\
p_{t-1}(\neq 0,0) & =: \operatorname{Pr}\left(\bar{Y}_{t-1}^{1} \neq 0, \bar{Y}_{t-1}^{0}=0\right) .
\end{aligned}
$$

We derive bounds on ATETS $_{t}$ defined by

$$
\mathbb{E}\left[Y_{t}^{1} \mid \bar{Y}_{t-1}^{1}=0\right]-\mathbb{E}\left[Y_{t}^{0} \mid \bar{Y}_{t-1}^{1}=0\right]
$$

with the data providing the observed transition probabilities $\operatorname{Pr}\left(Y_{t}=y_{t} \mid \bar{Y}_{t-1}=0, D=1\right)$ and $\operatorname{Pr}\left(Y_{t}=y_{t} \mid \bar{Y}_{t-1}=0, D=0\right)$. 
Under Assumption 1

$$
\mathbb{E}\left[Y_{t}^{1} \mid \bar{Y}_{t-1}^{1}=0\right]=\operatorname{Pr}\left(Y_{t}=1 \mid \bar{Y}_{t-1}=0, D=1\right),
$$

so that if $\operatorname{Pr}\left(\bar{Y}_{t-1}^{1}=0 \mid D=1\right)=\operatorname{Pr}\left(\bar{Y}_{t-1}=0 \mid D=1\right)>0$ then $\mathbb{E}\left[Y_{t}^{1} \mid \bar{Y}_{t-1}^{1}=0\right]$ is point-identified, and if $\operatorname{Pr}\left(\bar{Y}_{t-1}^{1}=0 \mid D=1\right)=\operatorname{Pr}\left(\bar{Y}_{t-1}=0 \mid D=1\right)=0$ then $\mathbb{E}\left[Y_{t}^{1} \mid \bar{Y}_{t-1}^{1}=0\right], \mathbb{E}\left[Y_{t}^{0} \mid \bar{Y}_{t-1}^{l-1}=0\right]$ and ATETS ${ }_{t}$ are not defined. Note that the point identification of this mean is similar to the point identification of the treated mean in the ATET in static settings.

Next, we have for the counterfactual transition probability

$$
\mathbb{E}\left[Y_{t}^{0} \mid \bar{Y}_{t-1}^{1}=0\right]=\frac{p_{t}^{0}(1 \mid 0,0) p_{t-1}(0,0)+p_{t}^{0}(1 \mid 0, \neq 0) p_{t-1}(0, \neq 0)}{p_{t-1}(0,0)+p_{t-1}(0, \neq 0)} .
$$

By Assumption 1

$$
\operatorname{Pr}\left(Y_{t}=1, \bar{Y}_{t-1}=0 \mid D=0\right)=\operatorname{Pr}\left(Y_{t}^{0}=1, \bar{Y}_{t-1}^{0}=0 \mid D=0\right)=\operatorname{Pr}\left(Y_{t}^{0}=1, \bar{Y}_{t-1}^{0}=0\right) .
$$

By the law of total probability

$$
\begin{aligned}
& \operatorname{Pr}\left(Y_{t}^{0}=1, \bar{Y}_{t-1}^{0}=0\right)=\operatorname{Pr}\left(\bar{Y}_{t-1}^{1}=0, Y_{t}^{0}=1, \bar{Y}_{t-1}^{0}=0\right)+\operatorname{Pr}\left(\bar{Y}_{t-1}^{1} \neq 0, Y_{t}^{0}=1, \bar{Y}_{t-1}^{0}=0\right)= \\
& p_{t}^{0}(1 \mid 0,0) p_{t-1}(0,0)+p_{t}^{0}(1 \mid \neq 0,0) p_{t-1}(\neq 0,0) .
\end{aligned}
$$

Therefore,

$$
\operatorname{Pr}\left(Y_{t}=1, \bar{Y}_{t-1}=0 \mid D=0\right)=p_{t}^{0}(1 \mid 0,0) p_{t-1}(0,0)+p_{t}^{0}(1 \mid \neq 0,0) p_{t-1}(\neq 0,0)
$$

Solving for $p_{t}^{0}(1 \mid 0,0)$ gives

$$
p_{t}^{0}(1 \mid 0,0)=\frac{\operatorname{Pr}\left(Y_{t}=1, \bar{Y}_{t-1}=0 \mid D=0\right)-p_{t}^{0}(1 \mid \neq 0,0) p_{t-1}(\neq 0,0)}{p_{t-1}(0,0)}
$$

and upon substitution

$$
\mathbb{E}\left[Y_{t}^{0} \mid \bar{Y}_{t-1}^{1}=0\right]=\frac{\operatorname{Pr}\left(Y_{t}=1, \bar{Y}_{t-1}=0 \mid D=0\right)}{p_{t-1}(0,0)+p_{t-1}(0, \neq 0)}-\frac{p_{t}^{0}(1 \mid \neq 0,0) p_{t-1}(\neq 0,0)-p_{t}^{0}(1 \mid 0, \neq 0) p_{t-1}(0, \neq 0)}{p_{t-1}(0,0)+p_{t-1}(0, \neq 0)} .
$$

The expression on the right-hand side is decreasing in $p_{t}^{0}(1 \mid \neq 0,0)$ and increasing in $p_{t}^{0}(1 \mid 0, \neq 0)$. The lower bound is obtained by setting $p_{t}^{0}(1 \mid \neq 0,0)$ at 1 and $p_{t}^{0}(1 \mid 0, \neq 0)$ at 0 and the upper bound by setting $p_{t}^{0}(1 \mid \neq 0,0)$ at 0 and $p_{t}^{0}(1 \mid 0, \neq 0)$ at 1 so that

$$
\begin{aligned}
& \frac{\operatorname{Pr}\left(Y_{t}=1 \mid \bar{Y}_{t-1}=0, D=0\right) \operatorname{Pr}\left(\bar{Y}_{t-1}=0 \mid D=0\right)-p_{t-1}(\neq 0,0)}{p_{t-1}(0,0)+p_{t-1}(0, \neq 0)} \\
& \quad \leq \mathbb{E}\left[Y_{t}^{0} \mid \bar{Y}_{t-1}^{1}=0\right] \leq \\
& \frac{\operatorname{Pr}\left(Y_{t}=1 \mid \bar{Y}_{t-1}=0, D=0\right) \operatorname{Pr}\left(\bar{Y}_{t-1}=0 \mid D=0\right)+p_{t-1}(0, \neq 0)}{p_{t-1}(0,0)+p_{t-1}(0, \neq 0)},
\end{aligned}
$$

where we note that

$$
\operatorname{Pr}\left(Y_{t}=1 \mid \bar{Y}_{t-1}=0, D=0\right) \operatorname{Pr}\left(\bar{Y}_{t-1}=0 \mid D=0\right)=\operatorname{Pr}\left(Y_{t}=1, \bar{Y}_{t-1}=0 \mid D=0\right)=0
$$

if $\operatorname{Pr}\left(\bar{Y}_{t-1}=0 \mid D=0\right)=0$.

Because

$$
\operatorname{Pr}\left(\bar{Y}_{t-1}=0 \mid D=1\right)=p_{t-1}(0,0)+p_{t-1}(0, \neq 0)
$$

and

$$
\operatorname{Pr}\left(\bar{Y}_{t-1}=0 \mid D=0\right)=p_{t-1}(0,0)+p_{t-1}(\neq 0,0)
$$

we have

$$
\begin{aligned}
& \frac{\left[\operatorname{Pr}\left(Y_{t}=1 \mid \bar{Y}_{t-1}=0, D=0\right)-1\right] \operatorname{Pr}\left(\bar{Y}_{t-1}=0 \mid D=0\right)+p_{t-1}(0,0)}{\operatorname{Pr}\left(\bar{Y}_{t-1}=0 \mid D=1\right)} \\
& \leq \mathbb{E}\left[Y_{t}^{0} \bar{Y}_{t-1}^{1}=0\right] \leq \\
& \frac{\operatorname{Pr}\left(Y_{t}=1 \mid \bar{Y}_{t-1}=0, D=0\right) \operatorname{Pr}\left(\bar{Y}_{t-1}=0 \mid D=0\right)-p_{t-1}(0,0)}{\operatorname{Pr}\left(\bar{Y}_{t-1}=0 \mid D=1\right)}+1 .
\end{aligned}
$$


The upper bound is decreasing and the lower bound is increasing in $p_{t-1}(0,0)$. By the Bonferroni inequality

$$
\begin{aligned}
& p_{t-1}(0,0) \geq \max \left\{\operatorname{Pr}\left(\bar{Y}_{t-1}^{1}=0\right)+\operatorname{Pr}\left(\bar{Y}_{t-1}^{0}=0\right)-1,0\right\}= \\
& \max \left\{\operatorname{Pr}\left(\bar{Y}_{t-1}=0 \mid D=1\right)+\operatorname{Pr}\left(\bar{Y}_{t-1}=0 \mid D=0\right)-1,0\right\} .
\end{aligned}
$$

If

$$
\operatorname{Pr}\left(\bar{Y}_{t-1}=0 \mid D=1\right)+\operatorname{Pr}\left(\bar{Y}_{t-1}=0 \mid D=0\right)-1 \leq 0
$$

the lower bound on $p_{t-1}(0,0)$ is 0 . In that case the lower bound in (A.3) is non-positive and the upper bound is greater than or equal to 1 so that

$$
0 \leq \mathbb{E}\left[Y_{t}^{0} \mid \bar{Y}_{t-1}^{1}=0\right] \leq 1 .
$$

If $\operatorname{Pr}\left(\bar{Y}_{t-1}=0 \mid D=1\right)+\operatorname{Pr}\left(\bar{Y}_{t-1}=0 \mid D=0\right)-1>0$ we have upon substitution of the lower bound on $p_{t-1}(0,0)$ into (A.3) and because the probability $\mathbb{E}\left[Y_{t}^{0} \mid \bar{Y}_{t-1}^{1}=0\right]$ is bounded by zero and one

$$
\begin{aligned}
& \max \left\{0, \frac{\operatorname{Pr}\left(Y_{t}=1 \mid \bar{Y}_{t-1}=0, D=0\right) \operatorname{Pr}\left(\bar{Y}_{t-1}=0 \mid D=0\right)-1}{\operatorname{Pr}\left(\bar{Y}_{t-1}=0 \mid D=1\right)}+1\right\} \\
& \leq \mathbb{E}\left[Y_{t}^{0} \mid \bar{Y}_{t-1}^{1}=0\right] \leq \\
& \min \left\{1, \frac{1-\left[1-\operatorname{Pr}\left(Y_{t}=1 \mid \bar{Y}_{t-1}=0, D=0\right)\right] \operatorname{Pr}\left(\bar{Y}_{t-1}=0 \mid D=0\right)}{\operatorname{Pr}\left(\bar{Y}_{t-1}=0 \mid D=1\right)}\right\} .
\end{aligned}
$$

Finally, we combine these bounds with the point-identified $\mathbb{E}\left[Y_{t}^{1} \mid \bar{Y}_{t-1}^{1}=0\right]$ to obtain bounds on ATETS .

\section{Proof of Theorem 2.}

As above, under Assumption $1 \mathbb{E}\left[Y_{t}^{1} \mid \bar{Y}_{t-1}^{1}=0\right]=\operatorname{Pr}\left(Y_{t}=1 \mid \bar{Y}_{t-1}=0, D=1\right)$, so that if $\operatorname{Pr}\left(\bar{Y}_{t-1}=0 \mid D=1\right)>0$ then $\mathbb{E}\left[Y_{t}^{1} \mid \bar{Y}_{t-1}^{1}=0\right]$ is point-identified, and if $\operatorname{Pr}\left(\bar{Y}_{t-1}=0 \mid D=1\right)=0$ then $\operatorname{ATETS}_{t}$ is not defined. If $\operatorname{Pr}\left(\bar{Y}_{t-1}=0 \mid D=1\right)>0$ we have from (A.3)

$$
\begin{aligned}
& \frac{\left[\operatorname{Pr}\left(Y_{t}=1 \mid \bar{Y}_{t-1}=0, D=0\right)-1\right] \operatorname{Pr}\left(\bar{Y}_{t-1}=0 \mid D=0\right)+p_{t-1}(0,0)}{\operatorname{Pr}\left(\bar{Y}_{t-1}=0 \mid D=1\right)} \\
& \leq \mathbb{E}\left[Y_{t}^{0} \mid \bar{Y}_{t-1}^{1}=0\right] \leq \\
& \frac{\operatorname{Pr}\left(Y_{t}=1 \mid \bar{Y}_{t-1}=0, D=0\right) \operatorname{Pr}\left(\bar{Y}_{t-1}=0 \mid D=0\right)-p_{t-1}(0,0)}{\operatorname{Pr}\left(\bar{Y}_{t-1}=0 \mid D=1\right)}+1 .
\end{aligned}
$$

Because the lower bound is increasing in $p_{t-1}(0,0)$ and the upper bound decreasing in $p_{t-1}(0,0)$ we need the lower bound on this probability. We have

$$
\begin{aligned}
& p_{t-1}(0,0)=\operatorname{Pr}\left(Y_{t-1}^{1}=0, \ldots, Y_{1}^{1}=0, Y_{t-1}^{0}=0, \ldots, Y_{1}^{0}=0\right)= \\
& \operatorname{Pr}\left(Y_{t-1}^{1}=0, Y_{t-1}^{0}=0 \mid S_{t-2}\right) \operatorname{Pr}\left(Y_{t-2}^{1}=0, \ldots, Y_{1}^{1}=0, Y_{t-2}^{0}=0, \ldots, Y_{1}^{0}=0\right) .
\end{aligned}
$$

By Assumption 3 either

$$
\operatorname{Pr}\left(Y_{t-1}^{1}=0 \mid S_{t-2}, V\right) \leq \operatorname{Pr}\left(Y_{t-1}^{0}=0 \mid S_{t-2}, V\right),
$$

or

$$
\operatorname{Pr}\left(Y_{t-1}^{1}=0 \mid S_{t-2}, V\right)>\operatorname{Pr}\left(Y_{t-1}^{0}=0 \mid S_{t-2}, V\right),
$$

for all $V$. Assume that (A.6) holds. By Assumption 4 this implies that

$$
\operatorname{Pr}\left(Y_{t-1}^{1}=0, Y_{t-1}^{0}=1 \mid S_{i, t-2}, V\right)=0,
$$


so that

$$
\begin{aligned}
\operatorname{Pr}\left(Y_{t-1}^{1}=0 \mid S_{t-2}, V\right) & =\operatorname{Pr}\left(Y_{t-1}^{1}=0, Y_{t-1}^{0}=0 \mid S_{t-2}, V\right)+\operatorname{Pr}\left(Y_{t-1}^{1}=0, Y_{t-1}^{0}=1 \mid S_{t-2}, V\right) \\
& =\operatorname{Pr}\left(Y_{t-1}^{1}=0, Y_{t-1}^{0}=0 \mid S_{t-2}, V\right) .
\end{aligned}
$$

Because this holds for all $V$ we omit $V$ in the sequel. Because Assumptions 3 and 4 hold for all $t$, it follows from this equation by recursion that

$$
\operatorname{Pr}\left(Y_{t-1}^{1}=0, \ldots, Y_{1}^{1}=0, Y_{t-1}^{0}=0, \ldots, Y_{1}^{0}=0\right)=\prod_{s=1}^{t-1} \operatorname{Pr}\left(Y_{s}^{1}=0 \mid \bar{Y}_{s-1}^{1}=0\right),
$$

so that

$$
p_{t-1}(0,0)=\prod_{s=1}^{t-1} \operatorname{Pr}\left(Y_{s}^{1}=0 \mid \bar{Y}_{s-1}^{1}=0\right)=\prod_{s=1}^{t-1} \operatorname{Pr}\left(Y_{s}=0 \mid \bar{Y}_{s-1}=0, D=1\right) .
$$

If Assumption 3 holds with (A.7), then

$$
p_{t-1}(0,0)=\prod_{s=1}^{t-1} \operatorname{Pr}\left(Y_{s}^{0}=0 \mid \bar{Y}_{s-1}^{0}=0\right)=\prod_{s=1}^{t-1} \operatorname{Pr}\left(Y_{s}=0 \mid \bar{Y}_{s-1}=0, D=0\right) .
$$

We conclude that

$$
\begin{aligned}
& p_{t-1}(0,0) \geq \min \left\{\prod_{s=1}^{t-1} \operatorname{Pr}\left(Y_{s}=0 \mid \bar{Y}_{s-1}=0, D=1\right), \prod_{s=1}^{t-1} \operatorname{Pr}\left(Y_{s}=0 \mid \bar{Y}_{s-1}=0, D=0\right)\right\}= \\
& \min \left\{\operatorname{Pr}\left(\bar{Y}_{t-1}=0 \mid D=1\right), \operatorname{Pr}\left(\bar{Y}_{t-1}=0 \mid D=0\right)\right\} .
\end{aligned}
$$

As noted below Theorem 2 the bounds simplifies in an obvious way if we have prior knowledge of the direction of the effect of the treatment.

Next, upon substitution of this lower bound on $p_{t-1}(0,0)$ into $(A .3)$ and because the probability $\mathbb{E}\left[Y_{t}^{0} \mid \bar{Y}_{t-1}^{1}=0\right]$ is bounded by zero and one we have

$$
\begin{gathered}
\max \left\{0, \frac{\left[\operatorname{Pr}\left(Y_{t}=1 \mid \bar{Y}_{t-1}=0, D=0\right)-1\right] \operatorname{Pr}\left(\bar{Y}_{t-1}=0 \mid D=0\right)}{\operatorname{Pr}\left(\bar{Y}_{t-1}=0 \mid D=1\right)}\right. \\
\left.+\frac{\min \left\{\operatorname{Pr}\left(\bar{Y}_{t-1}=0 \mid D=1\right) \operatorname{Pr}\left(\bar{Y}_{t-1}=0 \mid D=0\right)\right\}}{\operatorname{Pr}\left(\bar{Y}_{t-1}=0 \mid D=1\right)}\right\} . \\
\leq \mathbb{E}\left[Y_{t}^{0} \mid \bar{Y}_{t-1}^{1}=0\right] \leq \\
\min \left\{1,1+\frac{\operatorname{Pr}\left(Y_{t}=1 \mid \bar{Y}_{t-1}=0, D=0\right) \operatorname{Pr}\left(\bar{Y}_{t-1}=0 \mid D=0\right)}{\operatorname{Pr}\left(\bar{Y}_{t-1}=0 \mid D=1\right)}\right. \\
\left.-\frac{\min \left\{\operatorname{Pr}\left(\bar{Y}_{t-1}=0 \mid D=1\right), \operatorname{Pr}\left(\bar{Y}_{t-1}=0 \mid D=0\right)\right\}}{\operatorname{Pr}\left(\bar{Y}_{t-1}=0 \mid D=1\right)}\right\} .
\end{gathered}
$$

Finally, we combine these bounds with the point-identified $\mathbb{E}\left[Y_{t}^{1} \mid \bar{Y}_{t-1}^{1}=0\right]$ to obtain bounds on ATETS $_{t}$.

\section{Proof of Theorem 3.}

As above, under Assumption $1 \mathbb{E}\left[Y_{t}^{1} \mid \bar{Y}_{t-1}^{1}=0\right]=\operatorname{Pr}\left(Y_{t}=1 \mid \bar{Y}_{t-1}=0, D=1\right)$, so that if $\operatorname{Pr}\left(\bar{Y}_{t-1}=0 \mid D=1\right)>0$ then $\mathbb{E}\left[Y_{t}^{1} \mid \bar{Y}_{t-1}^{1}=0\right]$ is point-identified, and if $\operatorname{Pr}\left(\bar{Y}_{t-1}=0 \mid D=1\right)=0$ then $\operatorname{ATETS}_{t}$ is not defined.

Next, we have for the counterfactual transition probability

$$
\mathbb{E}\left[Y_{t}^{0} \mid \bar{Y}_{t-1}^{1}=0\right]=\frac{p_{t}^{0}(1 \mid 0,0) p_{t-1}(0,0)+p_{t}^{0}(1 \mid 0, \neq 0) p_{t-1}(0, \neq 0)}{p_{t-1}(0,0)+p_{t-1}(0, \neq 0)} .
$$

The expression on the right-hand side is increasing in $p_{t}^{0}(1 \mid 0, \neq 0)$. By Assumption 5 we have the restriction $p_{t}^{0}(1 \mid 0, \neq$ $0) \geq p_{t}^{0}(1 \mid 0,0)$. Then the upper bound is obtained by setting $p_{t}^{0}(1 \mid 0, \neq 0)=1$ and lower bound by setting $p_{t}^{0}(1 \mid 0, \neq 0)=$ 
$p_{t}^{0}(1 \mid 0,0)$ :

$$
p_{t}^{0}(1 \mid 0,0) \leq \mathbb{E}\left[Y_{t}^{0} \mid \bar{Y}_{t-1}^{1}=0\right] \leq \frac{p_{t}^{0}(1 \mid 0,0) p_{t-1}(0,0)+p_{t-1}(0, \neq 0)}{p_{t-1}(0,0)+p_{t-1}(0, \neq 0)} .
$$

By Assumption 1 and the law of total probability we have using similar reasoning as for Theorem 1 :

$$
\operatorname{Pr}\left(Y_{t}=1, \bar{Y}_{t-1}=0 \mid D=0\right)=p_{t}^{0}(1 \mid 0,0) p_{t-1}(0,0)+p_{t}^{0}(1 \mid \neq 0,0) p_{t-1}(\neq 0,0) .
$$

Solving for $p_{t}^{0}(1 \mid 0,0)$ gives

$$
p_{t}^{0}(1 \mid 0,0)=\frac{\operatorname{Pr}\left(Y_{t}=1, \bar{Y}_{t-1}=0 \mid D=0\right)-p_{t}^{0}(1 \mid \neq 0,0) p_{t-1}(\neq 0,0)}{p_{t-1}(0,0)}
$$

and upon substitution

$$
\begin{aligned}
& \frac{\operatorname{Pr}\left(Y_{t}=1, \bar{Y}_{t-1}=0 \mid D=0\right)-p_{t}^{0}(1 \mid \neq 0,0) p_{t-1}(\neq 0,0)}{p_{t-1}(0,0)} \leq \mathbb{E}\left[Y_{t}^{0} \mid \bar{Y}_{t-1}^{1}=0\right] \leq \\
& \frac{\operatorname{Pr}\left(Y_{t}=1, \bar{Y}_{t-1}=0 \mid D=0\right)-p_{t}^{0}(1 \mid \neq 0,0) p_{t-1}(\neq 0,0)+p_{t-1}(0, \neq 0)}{p_{t-1}(0,0)+p_{t-1}(0, \neq 0)} .
\end{aligned}
$$

Both the lower and upper bound is decreasing in $p_{t}^{0}(1 \mid \neq 0,0)$. By Assumption 5 we have the restriction $p_{t}^{0}(1 \mid \neq 0,0) \geq$ $p_{t}^{0}(1 \mid 0,0)$. Therefore the lower bound is obtained by setting $p_{t}^{0}(1 \mid \neq 0,0)$ at 1 . The upper bound is obtained by setting $p_{t}^{0}(1 \mid \neq 0,0)=p_{t}^{0}(1 \mid 0,0)$, upon substitution into (A.10) this implies that

$$
p_{t}^{0}(1 \mid \neq 0,0)=p_{t}^{0}(1 \mid 0,0)=\operatorname{Pr}\left(Y_{t}=1 \mid \bar{Y}_{t-1}=0, D=0\right) .
$$

Then,

$$
\begin{aligned}
& \frac{\operatorname{Pr}\left(Y_{t}=1, \bar{Y}_{t-1}=0 \mid D=0\right)-p_{t-1}(\neq 0,0)}{p_{t-1}(0,0)} \leq \mathbb{E}\left[Y_{t}^{0} \mid \bar{Y}_{t-1}^{1}=0\right] \leq \\
& \frac{\operatorname{Pr}\left(Y_{t}=1, \bar{Y}_{t-1}=0 \mid D=0\right)-\operatorname{Pr}\left(Y_{t}=1 \mid \bar{Y}_{t-1}=0, D=0\right) p_{t-1}(\neq 0,0)+p_{t-1}(0, \neq 0)}{p_{t-1}(0,0)+p_{t-1}(0, \neq 0)} .
\end{aligned}
$$

Because

$$
\begin{aligned}
& \operatorname{Pr}\left(\bar{Y}_{t-1}=0 \mid D=1\right)=p_{t-1}(0,0)+p_{t-1}(0, \neq 0) \\
& \operatorname{Pr}\left(\bar{Y}_{t-1}=0 \mid D=0\right)=p_{t-1}(0,0)+p_{t-1}(\neq 0,0)
\end{aligned}
$$

we have

$$
\begin{aligned}
& \frac{\operatorname{Pr}\left(Y_{t}=1, \bar{Y}_{t-1}=0 \mid D=0\right)-\operatorname{Pr}\left(\bar{Y}_{t-1}=0 \mid D=0\right)+p_{t-1}(0,0)}{p_{t-1}(0,0)} \\
& \quad \leq \mathbb{E}\left[Y_{t}^{0} \bar{Y}_{t-1}^{1}=0\right] \leq \frac{\left[\operatorname{Pr}\left(Y_{t}=1 \mid \bar{Y}_{t-1}=0, D=0\right)-1\right] p_{t-1}(0,0)}{\operatorname{Pr}\left(\bar{Y}_{t-1}=0 \mid D=1\right)}+1 .
\end{aligned}
$$

The lower bound is increasing and the upper bound decreasing in $p_{t-1}(0,0)$. Assumption 5 also improves on the Bonferroni inequality for $p_{t-1}(0,0)$. We have

$$
p_{t-1}(0,0)=\prod_{s=1}^{t-1} \operatorname{Pr}\left(Y_{s}^{1}=0, Y_{s}^{0}=0 \mid S_{s-1}\right)
$$

By the Bonferroni inequality and the results above

$$
\begin{aligned}
& \operatorname{Pr}\left(Y_{s}^{1}=0, Y_{s}^{0}=0 \mid S_{s-1}\right) \geq \max \left\{1-\operatorname{Pr}\left(Y_{s}^{1}=1 \mid S_{s-1}\right)-\operatorname{Pr}\left(Y_{s}^{0}=1 \mid S_{s-1}\right), 0\right\} \geq \\
& \max \left\{1-\operatorname{Pr}\left(Y_{s}=1 \mid \bar{Y}_{s-1}=0, D=1\right)-\operatorname{Pr}\left(Y_{s}=1 \mid \bar{Y}_{s-1}=0, D=0\right), 0\right\}= \\
& \max \left\{\operatorname{Pr}\left(Y_{s}=0 \mid \bar{Y}_{s-1}=0, D=1\right)+\operatorname{Pr}\left(Y_{s}=0 \mid \bar{Y}_{s-1}=0, D=0\right)-1,0\right\},
\end{aligned}
$$

so that

$$
p_{t-1}(0,0) \geq \prod_{s=1}^{t-1} \max \left\{\operatorname{Pr}\left(Y_{s}=0 \mid \bar{Y}_{s-1}=0, D=1\right)+\operatorname{Pr}\left(Y_{s}=0 \mid \bar{Y}_{s-1}=0, D=0\right)-1,0\right\} .
$$


We compare this to the lower bound

$$
\max \left\{\prod_{s=1}^{t-1} \operatorname{Pr}\left(Y_{s}=0 \mid \bar{Y}_{s-1}=0, D=1\right)+\prod_{s=1}^{t-1} \operatorname{Pr}\left(Y_{s}=0 \mid \bar{Y}_{s-1}=0, D=0\right)-1,0\right\}
$$

that we obtained in the proof of Theorem 1 . First, if there is an $1 \leq s^{\prime} \leq t-1$ so that

$$
\operatorname{Pr}\left(Y_{s^{\prime}}=0 \mid \bar{Y}_{s^{\prime}-1}=0, D=1\right)+\operatorname{Pr}\left(Y_{s^{\prime}}=0 \mid \bar{Y}_{s^{\prime}-1}=0, D=0\right)-1<0,
$$

then

$$
\begin{aligned}
& \prod_{s=1}^{t-1} \operatorname{Pr}\left(Y_{s}=0 \mid \bar{Y}_{s-1}=0, D=1\right)+\prod_{s=1}^{t-1} \operatorname{Pr}\left(Y_{s}=0 \mid \bar{Y}_{s-1}=0, D=0\right)-1= \\
& \operatorname{Pr}\left(Y_{s^{\prime}}=0 \mid \bar{Y}_{s^{\prime}-1}=0, D=1\right) \prod_{s=1, s \neq s^{\prime}}^{t-1} \operatorname{Pr}\left(Y_{s}=0 \mid \bar{Y}_{s-1}=0, D=1\right)+ \\
& \operatorname{Pr}\left(Y_{s^{\prime}}=0 \mid \bar{Y}_{s^{\prime}-1}=0, D=1\right) \prod_{s=1, s \neq s^{\prime}}^{t-1} \operatorname{Pr}\left(Y_{s}=0 \mid \bar{Y}_{s-1}=0, D=0\right)-1<0
\end{aligned}
$$

so that if the new lower bound is 0 , so is the previous one. Finally, if for all $s=1, \ldots, t-1$

$$
\operatorname{Pr}\left(Y_{s}=0 \mid \bar{Y}_{s-1}=0, D=1\right)+\operatorname{Pr}\left(Y_{s}=0 \mid \bar{Y}_{s-1}=0, D=0\right)-1>0,
$$

then

$$
\begin{aligned}
& \prod_{s=1}^{t-1}\left[\operatorname{Pr}\left(Y_{s}=0 \mid \bar{Y}_{s-1}=0, D=1\right)+\operatorname{Pr}\left(Y_{s}=0 \mid \bar{Y}_{s-1}=0, D=0\right)-1\right] \geq \\
& \prod_{s=1}^{t-1} \operatorname{Pr}\left(Y_{s}=0 \mid \bar{Y}_{s-1}=0, D=1\right)+\prod_{s=1}^{t-1} \operatorname{Pr}\left(Y_{s}=0 \mid \bar{Y}_{s-1}=0, D=0\right)-1 .
\end{aligned}
$$

If $\operatorname{Pr}\left(Y_{s}=0 \mid \bar{Y}_{s-1}=0, D=1\right)+\operatorname{Pr}\left(Y_{s}=0 \mid \bar{Y}_{s-1}=0, D=0\right)-1 \leq 0$ for some $s \leq t$ the lower bound on $p_{t-1}(0,0)$ is 0 . In that case the lower bound in (A.11) is non-positive and the upper bound is greater than or equal to 1 so that $0 \leq \mathbb{E}\left[Y_{t}^{0} \mid \bar{Y}_{t-1}^{1}=0\right] \leq 1$.

If $\operatorname{Pr}\left(Y_{s}=0 \mid \bar{Y}_{s-1}=0, \bar{D}_{s}=1\right)+\operatorname{Pr}\left(Y_{s}=0 \mid \bar{Y}_{s-1}=0, D=0\right)-1>0$ for all $s=1, \ldots, t-1$ we have upon substitution of the lower bound on $p_{t-1}(0,0)$ in (A.12) into (A.11) and because the probability $\mathbb{E}\left[Y_{t}^{0} \bar{Y}_{t-1}^{1}=0\right]$ is bounded by zero,

$$
\begin{aligned}
& \max \{0,\left.\frac{\left(\operatorname{Pr}\left(Y_{t}=1 \mid \bar{Y}_{t-1}=0, D=0\right)-1\right) \operatorname{Pr}\left(\bar{Y}_{t-1}=0 \mid D=0\right)}{\prod_{s=1}^{t-1}\left[\operatorname{Pr}\left(Y_{s}=0 \mid \bar{Y}_{s-1}=0, D=1\right)+\operatorname{Pr}\left(Y_{s}=0 \mid \bar{Y}_{s-1}=0, D=0\right)-1\right]}+1\right\} \\
& \leq \mathbb{E}\left[Y_{t}^{0} \mid \bar{Y}_{t-1}^{1}=0\right] \leq 1-\frac{1-\operatorname{Pr}\left(Y_{t}=1 \mid \bar{Y}_{t-1}=0, D=0\right)}{\operatorname{Pr}\left(\bar{Y}_{t-1}=0 \mid D=1\right)} . \\
& \cdot \prod_{s=1}^{t-1}\left[\operatorname{Pr}\left(Y_{s}=0 \mid \bar{Y}_{s-1}=0, D=1\right)+\operatorname{Pr}\left(Y_{s}=0 \mid \bar{Y}_{s-1}=0, D=0\right)-1\right] .
\end{aligned}
$$

Finally, we combine these bounds with the point-identified $\mathbb{E}\left[Y_{t}^{1} \mid \bar{Y}_{t-1}^{1}=0\right]$ to obtain bounds on ATETS .

\section{Proof of Theorem 4.}

Using similar reasoning as for the proof of Theorem 3 we have under Assumptions 1 and 5:

$$
\mathbb{E}\left[Y_{t}^{1} \mid \bar{Y}_{t-1}^{1}=0\right]=\operatorname{Pr}\left(Y_{t}=1 \mid \bar{Y}_{t-1}=0, D=1\right)
$$

and

$$
\begin{aligned}
& \frac{\operatorname{Pr}\left(Y_{t}=1, \bar{Y}_{t-1}=0 \mid D=0\right)-\operatorname{Pr}\left(\bar{Y}_{t-1}=0 \mid D=0\right)+p_{t-1}(0,0)}{p_{t-1}(0,0)} \\
& \quad \leq \mathbb{E}\left[Y_{t}^{0} \mid \bar{Y}_{t-1}^{1}=0\right] \leq \frac{\left[\operatorname{Pr}\left(Y_{t}=1 \mid \bar{Y}_{t-1}=0, D=0\right)-1\right] p_{t-1}(0,0)}{\operatorname{Pr}\left(\bar{Y}_{t-1}=0 \mid D=1\right)}+1 .
\end{aligned}
$$


The lower bound on $\mathbb{E}\left[Y_{t}^{0} \mid \bar{Y}_{t-1}^{1}=0\right]$ is increasing and the upper bound on $\mathbb{E}\left[Y_{t}^{0} \mid \bar{Y}_{t-1}^{1}=0\right]$ is decreasing in $p_{t-1}(0,0)$. By the proof of Theorem 2 we have under Assumptions 3 and 4

$$
p_{t-1}(0,0) \geq \min \left\{\operatorname{Pr}\left(\bar{Y}_{t-1}=0 \mid D=1\right), \operatorname{Pr}\left(\bar{Y}_{t-1}=0 \mid D=0\right)\right\},
$$

so that

$$
\begin{gathered}
\max \left\{0, \frac{\left(\operatorname{Pr}\left(Y_{t}=1 \mid \bar{Y}_{t-1}=0, D=0\right)-1\right) \operatorname{Pr}\left(\bar{Y}_{t-1}=0 \mid D=0\right)}{\min \left\{\operatorname{Pr}\left(\bar{Y}_{t-1}=0 \mid D=1\right), \operatorname{Pr}\left(\bar{Y}_{t-1}=0 \mid D=0\right)\right\}}+1\right\} \leq \mathbb{E}\left[Y_{t}^{0} \mid \bar{Y}_{t-1}^{1}=0\right] \leq \\
\frac{1-\operatorname{Pr}\left(Y_{t}=1 \mid \bar{Y}_{t-1}=0, D=0\right)}{\operatorname{Pr}\left(\bar{Y}_{t-1}=0 \mid D=1\right)} \times \min \left\{\operatorname{Pr}\left(\bar{Y}_{t-1}=0 \mid D=1\right), \operatorname{Pr}\left(\bar{Y}_{t-1}=0 \mid D=0\right)\right\}+1 .
\end{gathered}
$$

Together with the results for $\mathbb{E}\left[Y_{t}^{1} \mid \bar{Y}_{t-1}^{1}=0\right]$ this gives the bounds.

\section{Appendix B. Supplementary data}

\section{Supplementary material related to this article can be found online at https://doi.org/10.1016/j.jeconom.2017.11.012.}

\section{References}

Abbring, J.H., Heckman, J.J., 2007. Econometric evaluation of social programs, part III: Distributional treatment effects, dynamic treatment effects, dynamic discrete choice, and general equilibrium policy evaluation. In: Heckman, J., Leamer, E. (Eds.), Handbook of Econometrics, Vol. 6. North Holland.

Abbring, J.H., Van den Berg, G.J., 2003. The non-parametric identification of treatment effects in duration models. Econometrica 71, $1491-1517$.

Andrews, D.W., Barwick, P.J., 2012. Inference for parameters defined by moment inequalities: A recommended moment selection procedure. Econometrica 80 (6), 2805-2826.

Andrews, D.W., Guggenberger, P., 2009. Validity of subsampling and plug-in asymptotic inference for parameters defined by moment inequalities. Econometric Theory 25 (03), 669-709.

Andrews, D.W, Soares, G., 2010. Inference for parameters defined by moment inequalities using generalized moment selection. Econometrica 78 (1), 119157.

Bijwaard, G., Ridder, G., 2005. Correcting for selective compliance in a re-employment bonus experiment. J. Econometrics 125, 77-111.

Bonnal, L., Fougere, F., Serandon, A., 1997. Evaluating the impact of French employment policies on individual labour market histories. Rev. Econom. Stud. 64, 683-713.

Card, D., Sullivan, D., 1988. Measuring the effect of subsidized training programs on movements in and out of unemployment. Econometrica 56, 497-530.

Chernozhukov, V., Hong, H., Tamer, E., 2007. Estimation and confidence regions for parameter sets in econometric models1. Econometrica 75 (5), $1243-$ 1284.

Cox, D.R., 1972. Regression models and life-tables (with discussion). J. R. Stat. Soc. 34, 187-220.

Elbers, C., Ridder, G., 1982. True and spurious duration dependence: The identifiability of the proportional hazards model. Rev. Econom. Stud. 49, $402-411$.

Gill, R.D., Robins, J.M., 2001. Causal inference for complex longitudinal data: The continuous case. Ann. Statist. 29, 1785-1811.

Gritz, R.M., 1993. The impact of training on the frequency and duration of employment. J. Econometrics 57, 21-51.

Hahn, J., Ridder, G., 2014. Non-standard tests through a composite null and alternative in point-identified parameters. J. Econ. Methods 4, 1-28.

Ham, J.C., LaLonde, R.J., 1996. The effect of sample selection and initial conditions in duration models: Evidence from experimental data on training. Econometrica 64, 175-205.

Heckman, J., Navarro, S., 2007. Dynamic discrete choice and dynamic treatment effects. J. Econometrics 136, 341-396.

Kluve, J., Card, D., Fertig, M., Gora, M., Jacobi, L., Jensen, P., Leetmaa, R., Nima, L., Patacchini, E., Schaffner, S., Schmidt, C.M., Klaauw, B.v.D., Weber, A., 2007. Active Labor Market Policies in Europe: Performance and Perspectives. Springer.

Manski, C.F., 1997. Monotone treatment response. Econometrica 65, 1311-1334.

Manski, C.F., Pepper, J., 2000. Monotone instrumental variables: with an application to the returns to schooling. Econometrica 68, 115-136.

Meyer, B.D., 1996. What have we learned from the Illinois reemployment bonus experiment? J. Labor. Econ. 14, 26-51.

Murphy, S.A., 2005. An experimental design for the development of adaptive treatment strategies. Stat. Med. 24 (10), 1455-1481.

Murphy, S.A., Bingham, D., 2009. Screening experiments for developing dynamic treatment regimes. J. Amer. Statist. Assoc. 104 (485), $391-408$.

Ridder, G., 1986. An event history approach to the evaluation of training, recruitment and employment programmes. J. Appl. Econometrics 1, 109-126.

Romano, J.P., Shaikh, A.M., 2008. Inference for identifiable parameters in partially identified econometric models. J. Statist. Plann. Inference 138 (9), $2786-$ 2807.

Rosen, A.M., 2008. Confidence sets for partially identified parameters that satisfy a finite number of moment inequalities. J. Econometrics 146 (1), $107-117$.

Van den Berg, G.J., 1990. Nonstationarity in job search theory. Rev. Econom. Stud. 57, 255-277.

Van den Berg, G.J., 2001. Duration models: specification, identification and multiple durations. In: Heckman, J.J., Leamer, E.E. (Eds.), Handbook of Econometrics, Vol. 6. North-Holland.

Woodbury, S.A., Spiegelman, R.G., 1987. Bonusses to workers and employers to reduce unemployment: randomized trials in Illinois. Amer. Econ. Rev. 77 (4), 513-530. 